\title{
Stochasticity Triggers Activation of the S-phase Checkpoint Pathway in Budding Yeast
}

\author{
Peijie Zhou, ${ }^{1,2, *}$ Xin Gao, ${ }^{1,3, *}$ Xiaoli Li, ${ }^{4, *}$ Linxi $\mathrm{Li}^{1,3}$ Caoyuan Niu, ${ }^{4}$ Qi Ouyang $\odot,{ }^{1,3}$ \\ Huiqiang Lou, ${ }^{4, \dagger}$ Tiejun Li® ${ }^{2, \$}$ and Fangting Li $\oplus^{1,3,8}$ \\ ${ }^{1}$ Center for Quantitative Biology, Peking University, Beijing 100871, China \\ ${ }^{2}$ LMAM and School of Mathematical Sciences, Peking University, Beijing 100871, China \\ ${ }^{3}$ School of Physics, Peking University, Beijing 100871, China \\ ${ }^{4}$ State Key Laboratory of Agro-Biotechnology and Beijing Advanced Innovation Center for Food Nutrition \\ and Human Health, College of Biological Sciences, China Agricultural University, \\ Beijing, People's Republic of China
}

(Received 19 May 2020; revised 20 October 2020; accepted 17 November 2020; published 7 January 2021)

\begin{abstract}
In a complex and ever-changing environment, various signal transduction pathways mediate outside signals and stress to a living cell and its intracellular responses. Eukaryotic cells utilize the DNA synthesis phase (S-phase) checkpoint to respond to DNA damage and replication stress, and the activation of the S-phase checkpoint defers the routine progression in the S phase. Through the analysis of microfluidic single-cell measurements, we find that the behavior of yeast cells exhibits bimodal distribution in the activation of the S-phase checkpoint, and the nonactivated portion of cells obeys the exponential decay law over time, the rate of which is dictated by HU dosage. Mathematical modeling and further experimental evidence from different mutant strains support the idea that the activation of the yeast S-phase checkpoint is a stochastic barrier-crossing process in a double-well system, where the barrier height is determined by both DNA replication stress and autophosphorylation of the key effector kinase Rad53. Our approach, as a novel methodology, is generally applicable to quantitative analysis of the signal transduction pathways at the single-cell level.
\end{abstract}

DOI: 10.1103/PhysRevX.11.011004

Subject Areas: Biological Physics, Nonlinear Dynamics

\section{INTRODUCTION}

In a highly complex and volatile environment, living cells respond to different signals and execute corresponding cellular responses through signal transduction pathways and genetic regulatory networks. Different feedback loops exist in the signaling pathways and networks, resulting in nonlinearity that gives rise to various dynamic characteristics, such as ultrasensitivity, bistability, and periodic oscillations [1,2]. Among a population of clonal cells, random fluctuations in the environment and intracellular stochastic chemical reactions produce variability in behavior and variation of states [3]. The role of noise has been

\footnotetext{
${ }^{*}$ These authors contribute equally to this work.

Corresponding author.

lou@cau.edu.cn

*Corresponding author.

tieli@math.pku.edu.cn

${ }^{\S}$ Corresponding author. lft@pku.edu.cn

Published by the American Physical Society under the terms of the Creative Commons Attribution 4.0 International license. Further distribution of this work must maintain attribution to the author(s) and the published article's title, journal citation, and DOI.
}

analyzed from both experimental and theoretical aspects in gene expression [4-6], cell development [7-9], cell death $[10,11]$, and signal transduction $[12,13]$.

Understanding the role of noise in signal transduction pathways is important to study the cell-to-cell variability in cell signaling response to different types and levels of stress signals. Proposed models include stochastic dynamics perturbed by extrinsic noises from the external environment and intrinsic fluctuations of biochemical reactions in cells themselves, or deterministic evolution to different predetermined cellular states from different random initial states [12-14]. Two typical patterns, the graded or binary responses [15], arise naturally according to these two model types [Fig. 1(a)]. The graded response prefers predetermined cellular fates. Assume that the population of inactive cells initially forms a single peak around a low level of response. After activation by the stimulatory signal, they collectively relax towards a high level of response with a unimodal distribution. The dynamic system has a single steady state that shifts in response to its environment. In such circumstances, the noise is mainly embodied as a small fluctuation of parameters over the cells $[12,13]$. The binary response emphasizes the intrinsic noise of chemical reactions in cells. The activation process of the signal transduction pathway is usually depicted as the transition of individuals from the inactive state to the active state, 
(a)
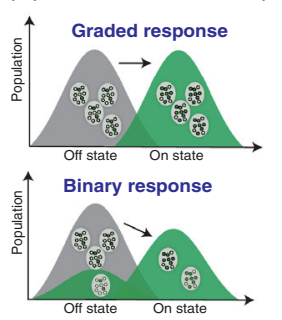

(b)

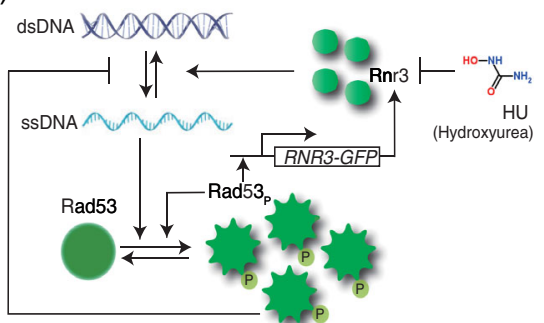

(c)

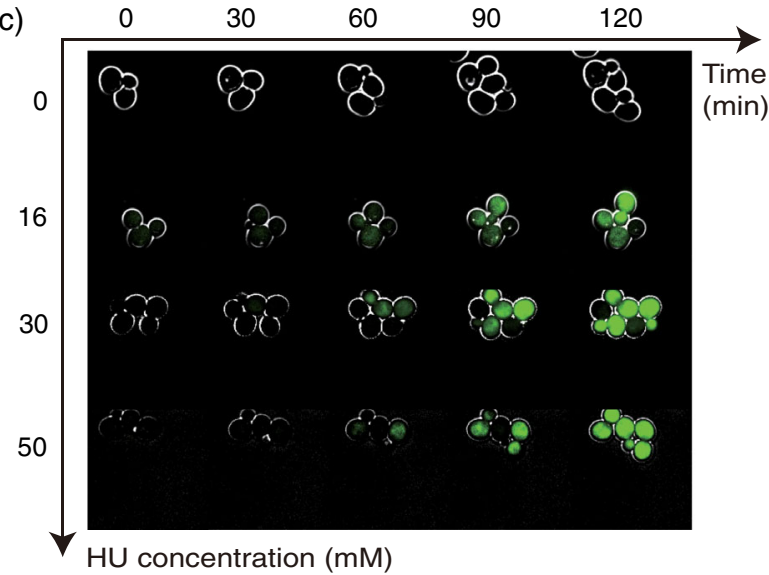

FIG. 1. Role of noise in the activation process of cell signal transduction pathways and the single-cell level experiment of hydroxyurea-induced S-phase checkpoint activation in budding yeast. (a) Two typical mechanisms of the activation process in cell signal transduction pathways involving stochastic noise. The activation process of a population of cells may be realized by the graded response whereby the key kinase level in all cells increases, together with a single peak, or the binary response whereby only a portion of cells undergo the switchlike transitions of states with a bimodal distribution over time. (b) Schematic regulatory network that governs the activation of the S-phase checkpoint triggered by HU stress in budding yeast. After DNA damage occurs, upstream signaling promotes phosphorylation of Rad53, a key kinase in the S-phase checkpoint, as well as its autophosphorylation. Phosphorylated Rad53 molecules activate the downstream kinase Dun 1 and then significantly enhance the transcription of Rnr3, which is marked with green fluorescence protein (GFP) in the single-cell experiment. This design allows us to determine the activation level of Rad53 by the fluorescence intensity of GFP. (c) Time-lapse fluorescence images of budding yeast WT strain cells in response to different doses of $\mathrm{HU}(0,16,30$, and $50 \mathrm{mM})$. Time from exposure of yeast cells to HU treatment was tracked.

which is mainly a barrier-crossing process assisted by the presence of noise $[16,17]$. From this perspective, the noise aids and triggers the hopping between metastable states, resulting in the evolution of bimodal distributions over time. Despite the important state switching evidence from gene expression systems $[18,19]$, the dynamical process of binary response in signaling the transduction pathway on the single-cell trajectory level remains to be explored.

In this work, we utilized the budding yeast Saccharomyces cerevisiae to study the S-phase checkpoint activation process at the single-cell level. It is known that the S-phase checkpoint in eukaryotic cells is a cellular response to DNA replication stress, and it can defer, or even block, the next progression in the DNA synthesis phase (S phase) in the cell cycle once it is activated under replication stress. In this sense, the S-phase checkpoint serves as the cell's surveillance system, which maintains DNA replication and genome integrity $[20,21]$. In budding yeast, the molecular regulatory mechanism of the S-phase checkpoint is one of the best-studied pathways [see Fig. 1(b) and more details in the next section]. However, its activation pattern, in the sense of the above two transition modes, is not fully understood.

It is thus interesting to investigate the mechanism of dynamic activation of the S-phase checkpoint (ASPC) of budding yeast. For this purpose, we treated the budding yeast BY4741 strain (called wild type, or WT) and key kinase rad53 mutants marked with Rnr3-GFP reporter [22] with various hydroxyurea (HU) doses (DNA replication stress) to induce the S-phase checkpoint, and we analyzed the time-lapse fluorescence results at the single-cell level with microscopy and microfluidic devices [Fig. 1(c)]. Experimentally, we selected the $s m l 1 \Delta$ strain as the control group since the knockout of the $S M L 1$ gene is necessary for the construction of rad53 mutants (see the Appendix A). By analyzing the heterogeneous response of budding yeast cells toward DNA replication stress, we found that the dynamics of S-phase checkpoint activation is a switchlike process with transitions from inactive to active states and that the population of inactivated cells obeys an exponential decay law over time, the rate of which is dictated by HU dosage. We then constructed both deterministic and stochastic models to explain the observed emergent dynamic behaviors. The theoretical prediction based on the proposed model and further experimental evidence from corresponding rad53 mutants supports a simple barrier-crossing mechanism in the activation process of the S-phase checkpoint. Our results show that both the DNA replication stress and the autophosphorylation of the key effector kinase Rad53 play an important role in the activation of the budding yeast S-phase checkpoint, while stochasticity triggers the activation of the $S$ phase in individual yeast cells.

\section{OVERVIEW OF ACTIVATION OF THE S-PHASE CHECKPOINT IN BUDDING YEAST}

In eukaryotic cells, faithful DNA replication is essential for genome stability and reproduction of cells. In replication, the signal transduction pathway is evolutionarily conserved from yeast to human cells, including sensor, adaptor, and effector kinase proteins. The key kinases of the S-phase checkpoint are Chk1/Rad53 in budding yeast and CHK1/CHK2 in human cells [23-25]. It is known that Rad53 ${ }^{\text {CHK2 }}$ plays a central role in the S-phase checkpoint to prevent genome rearrangement and cancer [26].

In budding yeast, the S-phase checkpoint can be triggered by various stress signals, including the lack of 
deoxyribonucleoside triphosphates (dNTPs), replication errors caused by DNA polymerases, and damage to DNA molecular structures [27,28]. HU is a ribonucleotide reductase (RNR) inhibitor. It is widely utilized in cell-cycle research to induce the activation process of the S-phase checkpoint in budding yeast by inducing DNA replication stress $[29,30]$. HU inhibits RNR activity and leads to the insufficiency dNTPs and subsequent DNA replication stalling $[31,32]$. At the stalled replication forks, the replication protein A (RPA), which wraps the accumulated singlestranded DNA (ssDNA), will recruit Mec1 and initiate signaling in the S-phase checkpoint pathway. The recruited Mec1 phosphorylates the key effector kinase Rad53 via the adaptors Mrc1 and Rad9 [33-37]. Subsequent autophosphorylation of Rad53 further increases the proportion of phosphorylated Rad53 molecules. These activated Rad53 molecules, in turn, phosphorylate and activate the downstream serine/threonine kinase Dun1 [38], the active form of which enhances the transcription level of $R N R 2 / 3 / 4$ via phosphorylating the transcriptional repressor Crt1 [39-42], thus promoting DNA replication fork stability and efficient recovery of DNA replication. When DNA damage is repaired, cells can resume cell-cycle progression after the activation process called checkpoint recovery. The S-phase checkpoint recovery process inactivates the upstream ssDNA signal and dephosphorylates Rad53 by the PP2C-like phosphatases before restarting DNA replication [43,44].

Overall, in an individual yeast cell, the DNA damage causes the accumulated ssDNA signal and thus triggers the massive phosphorylation of effector kinase Rad53 and transcription and translation of Rnr3, i.e., the S-phase checkpoint activation process [23]. After DNA damage is repaired, the ssDNA signal is decreased with the massive dephosphorylation of Rad53 in the yeast cell, corresponding to the S-phase checkpoint recovery process. The simplified S-phase checkpoint regulation network is schematically shown in Fig. 1(b), which focuses on $\operatorname{Rad} 53$ phosphorylation, the transcription and translation of Rnr3, and the arrest of the DNA replication fork. In this work, we only focus on the S-phase checkpoint activation process.

As discussed above, when most Rad53 molecules in a single yeast cell have been phosphorylated, the S-phase checkpoint in the cell should be activated and turned on, followed by the arrest of the DNA replication fork and the transcription and translation of Rnr3 protein. We simply say that the cell is in the on state of the S-phase checkpoint in such a case. Otherwise, we say that the cell is in the off state. For brevity, we abbreviate the activation of the S-phase checkpoint as ASPC and simply use "yeast cell" to indicate the budding yeast cell by default in later text.

\section{YEAST CELLS RESPOND HETEROGENEOUSLY TO STRESS IN S-PHASE CHECKPOINT ACTIVATION}

We utilized the time-lapse microscope to observe the activation process of the yeast S-phase checkpoint in single-cell resolution under DNA replication stress caused by different HU concentrations. However, in each living yeast cell, it is difficult to probe the effective Rad53 kinase directly or its phosphorylation state $(\operatorname{Rad} 53 \mathrm{P})$. In Fig. 2(a), we propose an indirect approach to infer the dynamics of the level of Rad53p. The protein Rnr3, the synthesis of which is regulated by Rad53p via transcriptional effects, is marked with GFP. Thus, the intensity of Rnr3 protein can be dynamically tracked by time-lapse fluorescence imaging in each single cell. We tracked each yeast cell and obtained the dynamic level of Rnr3 protein by time-lapse fluorescence imaging with 5-min time resolution. When most Rad53 molecules in a single yeast cell have been phosphorylated, the activation of the checkpoint will be followed by massive transcription and translation of Rnr3 protein. Hence, it is reasonable to characterize the Rad53p level, which is the Rnr3 promoter, by the synthesis rate of Rnr3. As shown in the middle panel of Fig. 2(a), we adopted piecewise linear regression for the Rnr3 fluorescence time series, and we utilized the fitted slopes as the estimated Rnr3 fluorescence growth rate (FGR) to approximate the dynamic level of Rad53p (see Sec. III of the Supplemental Material [45]). Local linear regression ensures better accuracy than the simple derivative estimate by the difference quotient.

Under different HU concentrations (8, 16, 30, 40, 50, 80, and $120 \mathrm{mM}$ ), we gathered the time-course data of Rnr3 fluorescence of 100-200 WT yeast cells for each time point (see Sec. IV. 1 of the Supplemental Material [45]) and processed their fluorescence growth rate. It is worth noting that although WT strain may be activated to the on state in an environment of $0-\mathrm{mM} \mathrm{HU}$, the possibility of activation for the first 200 cells during our 60 -min observation time is extremely low, so we ignored the effect of spontaneous DNA damage [46]. We found that cell-to-cell variability exists in these data. Intuitively, the response behavior of budding yeast cells toward HU stimulation can be classified into two groups. The first group of cells experiences an obvious increase in Rnr3 level, corresponding to a remarkable escalation in FGR, which indicates the massive phosphorylation of effector kinase Rad53 and the activation process of the S-phase checkpoint; e.g., see Fig. 2(a) when $\mathrm{HU}=80 \mathrm{mM}$. In these cells, after FGR reaches its peak, a declining trend follows, owing to the S-phase checkpoint recovery process, i.e., the DNA repair triggered by $\operatorname{Rad} 53$ p, which formulates a negative feedback to reduce the quantity of ssDNA and decrease the strength of the DNA replication stress. By contrast, the second group of cells maintains a relatively low synthetic level of Rnr3, implying the lower level of $\operatorname{Rad} 53 p$ and the failure to activate the S-phase checkpoint.

In order to identify the separation boundary between these two groups of cells quantitatively, it is necessary to quantify the extent of response in the S-phase checkpoint activation. Since we mainly focused on the activation 


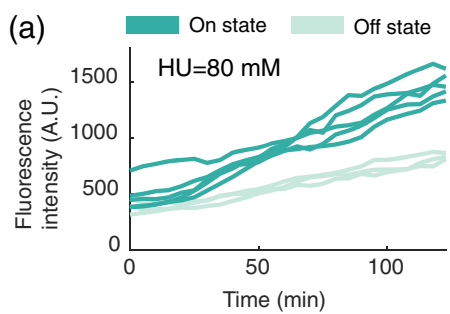

(c)
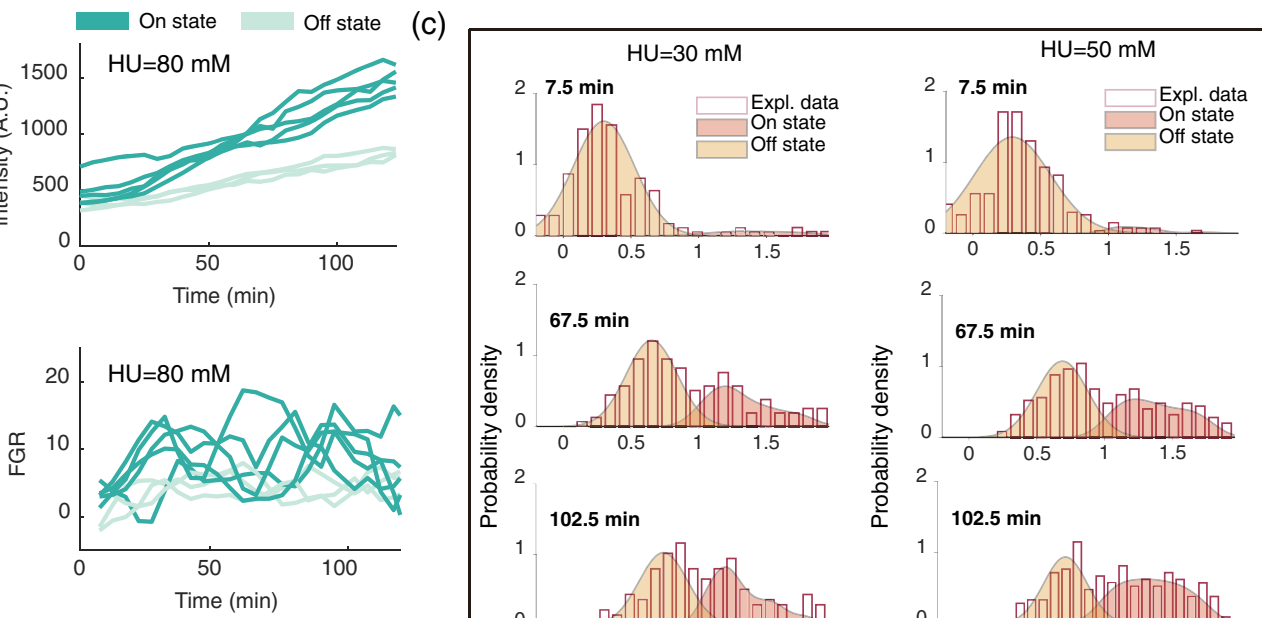

HU Concentration (mM)
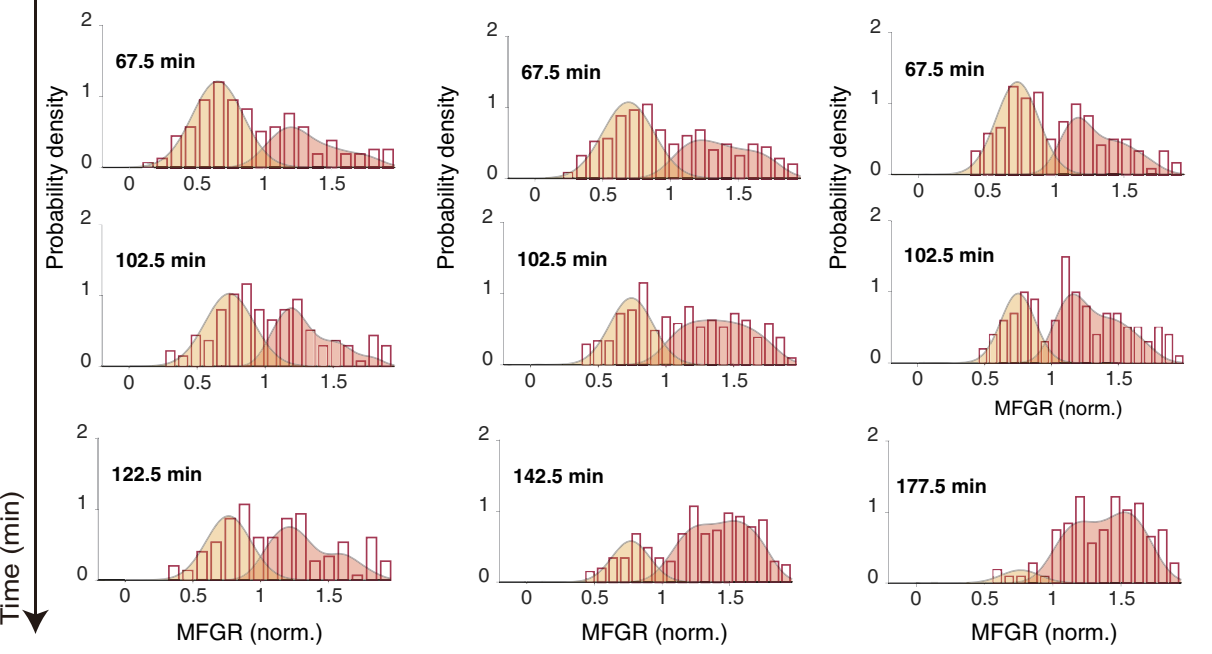

(b)
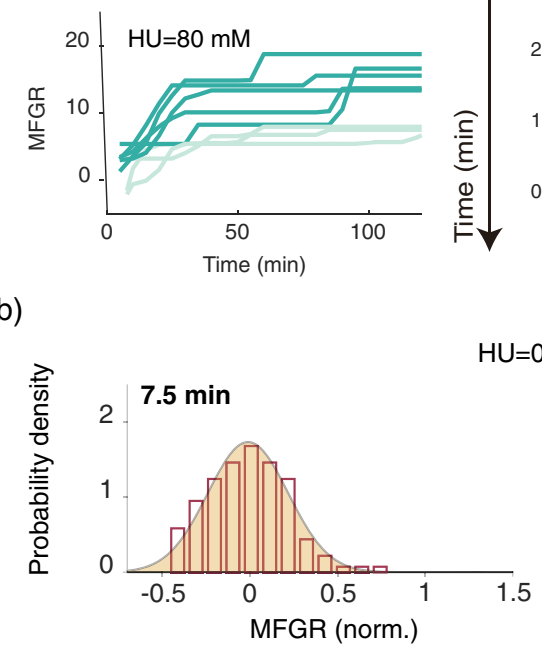

$\mathrm{HU}=0 \mathrm{mM}$

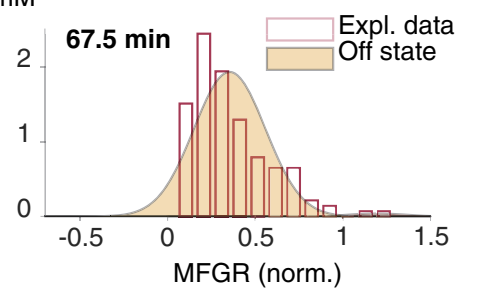

(d)

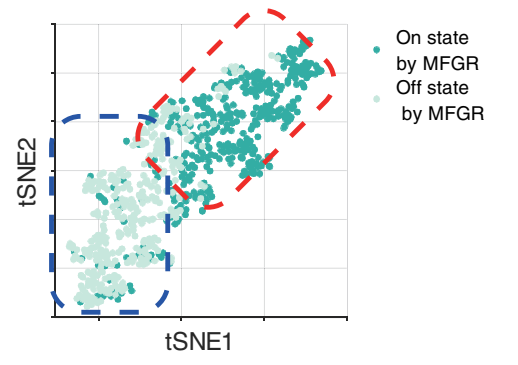

FIG. 2. Time series and analysis by the maximum value of fluorescence growth rate (MFGR) of Rnr3 protein and t-SNE plots for the original data, revealing the bimodal distribution of cellular states, corresponding to the inactivation/off and activation/on states of the Sphase checkpoint in yeast WT strain. (a) Some typical fluorescence time series in yeast cells under $\mathrm{HU}=80 \mathrm{mM}$ (top) and their corresponding fluorescence growth rates $F G R(t)$ (middle) and $M F G R(t)$ (bottom). Each trajectory represents the data from one individual cell, and its color represents the activation state, as determined from the bimodal distribution in Fig. 2(c). The time is initialized for each cell, starting from its first budding time. (b) Probability density distribution without HU treatment. It should be noted that the cells that have negative values at time $7.5 \mathrm{~min}$ will increase to positive values at time $67.5 \mathrm{~min}$, owing to the fluctuation of FGR, which does not mean the activation of these cells. (c) Probability density distribution of yeast WT strains for 30, 50, and 80-mM HU concentrations at various times. Compared with the control group (without $\mathrm{HU}$ treatment, $\mathrm{HU}=0 \mathrm{mM}$ ), The HU-treated groups evolve towards the bimodal distribution from the initial unimodal distribution, suggesting the existence of two different responsive states to the DNA replication stress signal. The off-state cells are fitted with Gaussian distribution based on the data (light yellow area), and on-state cells are fitted with kernel distribution (dark yellow area). (d) Raw data of the Rnr3 GFP time series with various HU doses (30, 50, and $80 \mathrm{mM}$ ), processed by the t-SNE dimensionality reduction method. The dark green points represent the activation state determined from Fig. 2(c). The low-dimension manifold of the time series can be clustered into two groups (marked by red dashed circles), corresponding to off and on states, respectively.

process of the S-phase checkpoint in this study, while ignoring the S-phase checkpoint recovery process that caused the decline of FGR, the maximum value of FGR was of particular interest. For a certain yeast cell, when the cell starts to bud at the beginning of the $S$ phase, we set $t=0$. Then, we calculated the maximum fluorescence growth rate of Rnr3 before time $t$, denoted as $\operatorname{MFGR}(t)$, which reflects the intensity of S-phase checkpoint activation until time $t$ from the beginning of the $\mathrm{S}$ phase. Thus, in a certain yeast cell, its FGR represents the activation as well as the deactivation (recovery) processes of the S-phase checkpoint, while its MFGR is a better indicator of whether the S-phase checkpoint has been activated (see Sec. III. 1 of the Supplemental Material [45]). In Fig. 2(a), we illustrate the original fluorescence of Rnr3, FGR, and MFGR from top to bottom, and the activated and inactive cells at each moment 
can be clearly distinguished by comparing MFGR instead of FGR.

\section{BIMODAL DISTRIBUTION OF MFGR OF RNR3 REVEALS ON AND OFF STATES OF S-PHASE CHECKPOINT ACTIVATION}

The distributions of MFGR $(t)$ under different HU doses at different time points are analyzed and listed in Sec. IV of the Supplemental Material [45], where we clearly observe the bimodal distribution of $\operatorname{MFGR}(t)$.

In Fig. 2(c), we illustrate the distribution of $\operatorname{MFGR}(t)$ under $\mathrm{HU}=30,50$, and $80 \mathrm{mM}$ at different times, respectively. When $\mathrm{HU}$ stress is absent $[\mathrm{HU}=0 \mathrm{mM}$, Fig. 2(b)], the probability density of $\operatorname{MFGR}(t)$ forms a unimodal distribution, and the unimodality remains over time. Because of random fluctuations, the distribution assumes nonzero probability for negative MFGR at $7.5 \mathrm{~min}$, and it shifts rightward at later times, e.g., the distribution at $67.5 \mathrm{~min}$ in Fig. 2(c); however, no cells are activated in the S-phase checkpoint. Under higher stress of $\mathrm{HU}(\mathrm{HU}=30,50$, and $80 \mathrm{mM})$, the distribution of MFGR at $7.5 \mathrm{~min}$ is still unimodal, but it gradually becomes bimodal with a new peak centered at a greater value of MFGR. As HU dosage increases, the percentage of cells with a higher MFGR value rises, suggesting that more cells are activated in the S-phase checkpoint.

The bimodal distribution of MFGR in Fig. 2(c) further elucidates the feature of cell-to-cell variability in S-phase checkpoint activation. When $\mathrm{HU}=0 \mathrm{mM}$, no DNA replication stress is induced by $\mathrm{HU}$; therefore, the checkpoint will not be activated, and the effector kinase number will fluctuate around a relatively low level in all cells. Hence, we observe the unimodal low level of $\operatorname{MFGR}(t)$ distribution in the control group without HU stress. Under higher stress of $\mathrm{HU}$, the checkpoint activation process happens heterogeneously among different cells, owing to the effect of noise. Within a certain time, a subpopulation of cells experience a drastic increase in $\operatorname{Rad} 53 \mathrm{p}$ level, the $\operatorname{MFGR}(t)$ of which forms a new peak in the histogram, while the remaining yeast cells remain inactivated and their $\operatorname{MFGR}(t)$ distribution stays around the original lower level peak, resulting in bimodality in $\operatorname{MFGR}(t)$ distribution, as shown in Fig. 2(c). Therefore, two peaks of $\operatorname{MFGR}(t)$ distribution correspond to two typical response patterns and cellular states. The cells that lie in the right part of the $\operatorname{MFGR}(t)$ distribution with higher Rad53p level are in the on state of the S-phase checkpoint, while the remaining cells in the left part of the MFGR distribution with lower Rad53p level are in the off state of the S-phase checkpoint. This result strongly supports the idea that the role of noise operates in accordance with the binary response, instead of the graded response [Fig. 1(a)], in the activation process of the yeast S-phase checkpoint.

The above observations are also validated by unsupervised dimensional reduction analysis to the original time series of Rnr3 GFP data from a pure data-driven perspective. We utilized t-distributed stochastic neighbor embedding (t-SNE) to visualize the original raw Rnr3 GFP fluorescence time series [Fig. 2(d)] and discovered two clusters on the data manifold that correspond to the on and off states of MFGR distribution. Specifically, the on-state cluster has a larger proportion of cells treated with higher doses of HU, suggesting the higher activation level of the Sphase checkpoint with the increase of DNA replication stress (see Sec. IV. 1 of the Supplemental Material [45]).

\section{ACTIVATION OF S-PHASE CHECKPOINT IN YEAST CELLS: DYNAMIC SWITCHING EVENT FROM OFF TO ON STATE}

We further investigate the dynamic properties of $\operatorname{MFGR}(t)$ distribution of Rnr3 protein in the WT strain under various doses of HU stress. Our results suggest that the ASPC in each single yeast cell is a dynamic switching event from off to on states.

First, we study how the distribution of $\operatorname{MFGR}(t)$ evolves dynamically over time. As time increases, according to the definition of MFGR, a rightward trend of probability distributions is expected to occur. In Fig. 3(a), we investigate the time dependence of the $\operatorname{MFGR}(t)$ histograms for hundreds of WT cells under the treatment of $80-\mathrm{mM} \mathrm{HU}$. With the increase of time, we observe that the bimodality of the $\operatorname{MFGR}(t)$ distribution arises and evolves with the increasing proportion of on-state cells and fewer off-state cells, while the position of their division line remains nearly invariant. In other words, the rightward trend of $\operatorname{MFGR}(t)$ bimodal distribution occurs by the decrease of the left peak and the increase of the right peak, instead of the direct translation of the whole distribution. Under a certain $\mathrm{HU}$ stress signal, then, more yeast cells are triggered from off to on states in S-phase checkpoint activation. This result supports the rationale of classifying cells into two groups according to bimodal distribution, and it further indicates that some cells would experience off-to-on state transitions with the induction of HU. Therefore, S-phase checkpoint activation can be regarded as a switching event from off to on states in response to the DNA replication stress signal.

As a consequence of the stochastic effect, the switching time from off to on states for a certain cell, denoted by $T_{\mathrm{SW}}$, is a random variable for each activated cell. A quantitative step is then taken to explore the dynamics of such switching from a survival analysis perspective [47]. At each time point $t$, the proportion of off-state cells, denoted by $R_{\text {off }}(t)$, can be calculated from the current $\operatorname{MFGR}(t)$ distribution by counting the ratio of the left cluster of cells-i.e., the inactive and nontriggered cells with lower MFGR level until time $t$ - to the whole population. In addition, $R_{\text {off }}(t)$ is the probability that the off-to-on switching time $T_{\mathrm{SW}}$ of a cell is greater than time $t$.

For WT strain under different HU concentrations, we plot in Fig. 3(b) the time evolution of $R_{\text {off }}(t)$ at the 
(a)

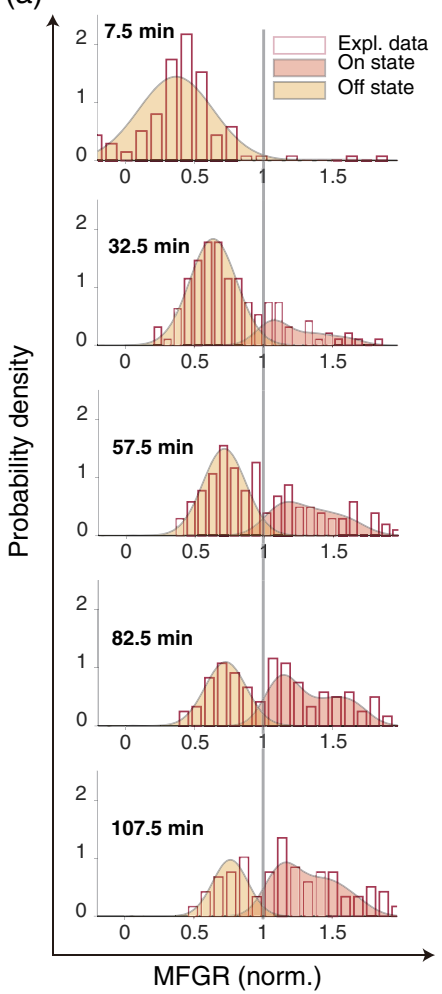

(b)

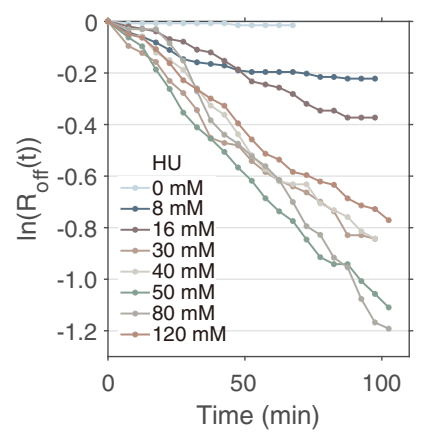

(e)

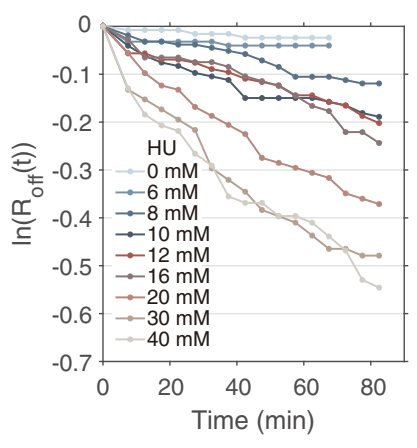

(c)

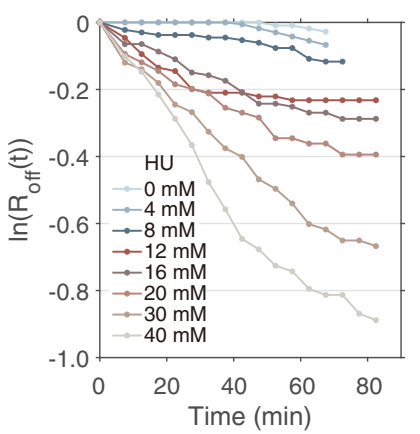

(f)

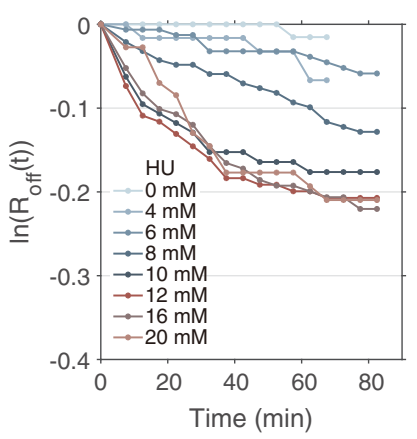

(d)

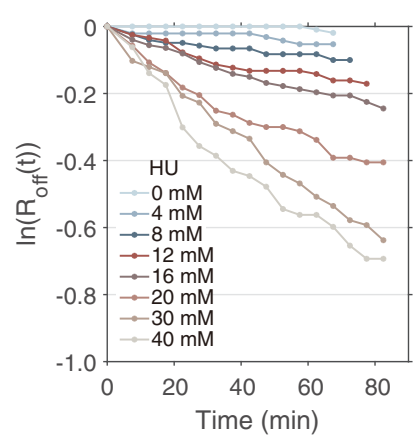

(g) Piecewise fitting (sm/1 $\Delta)$

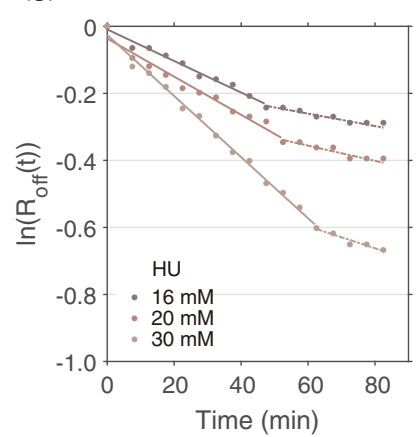

FIG. 3. Dynamic properties of MFGR of Rnr3 protein with respect to DNA replication stress in WT, sml1 $\Delta$, rad53-S350A, rad53-T354A, and rad53-SATA strains. (a) Bimodal probability distribution of maximum Rnr3 growth rate for $\mathrm{HU}=80 \mathrm{mM}$ at different times in WT yeast cells. As time evolves, the proportion of off-state cells decreases while the proportion of on-state cells increases, indicating the dynamic transition from off to on states. (b)-(f) Inactivation curve for different HU dosages in WT (b), sml1 $\Delta$ (c), rad53-S350A (d), rad53-T354A (e), and rad53-SATA (f) mutants. The $y$ label denotes the logarithm of the off-cell proportions, and the $x$ label denotes time, forming the inactivation curves for the DNA replication-stress-responsive switching process. The curve consists of a linear region and a constant region, implying the exponentially distributed stochastic responsive switching in the $\mathrm{S}$ phase and the termination of switching after the $\mathrm{S}$ phase. With the increase of HU concentration, the switching rate increases. It also suggests that the S-phase period of yeast cells under low HU concentrations is shorter than that of the high HU concentrations. (g) Linear decay (indicated by solid lines) and stationary regime (indicated by dotted lines) by piecewise linear fitting in $s m l 1 \Delta$ strains.

logarithmic scale. We find that each inactivation curve $f(t)=\ln R_{\text {off }}(t)$ consists of a linear decay region and a constant region [Fig. 3(g)]. In the linear decay region, as time evolves, the increasing proportion of yeast cells is activated from the off to on states. In the constant region, no more cells are activated to the on state of the S-phase checkpoint, suggesting that the cells have completed their S-phase process and that the proportion of off-state cells remains almost invariant. From Fig. 3(b), we also observe that the linear region of survival curves expands when $\mathrm{HU}$ concentration is increased, which is in agreement with our observation that the increase of $\mathrm{HU}$ concentration results in the elongation of the S-phase period in the yeast cell cycle (see Sec. III. 2 of the Supplemental Material [45]).

Then, we focus on the linear decay region of the $R_{\text {off }}(t)$ curves in Fig. 3(b). For a certain HU concentration, assuming that the slope of the linear region is $-\lambda$, we have $R_{\text {off }}(t)=P\left(T_{\mathrm{SW}}>T\right)=e^{-\lambda t}$, which suggests that the switching time $T_{\mathrm{SW}}$ is nearly exponentially distributed.
The switching rate $\lambda$, denoted as the switching rate from off to on states in ASPC, can be estimated by fitting the slopes of the linear region (see Sec. II of the Supplemental Material [45] for methods). Within the low HU concentration range ( $\mathrm{HU}<40 \mathrm{mM}$ ), we discover that an increase of $\mathrm{HU}$ concentration results in an increase of the switching rate $\lambda$, which suggests that a higher $\mathrm{HU}$ dose causes a stronger DNA replication stress, in turn boosting a larger activation rate of the S-phase checkpoint in yeast cells. On the other hand, in the high $\mathrm{HU}$ concentration range $(\mathrm{HU}>40 \mathrm{mM})$, such boosting displays saturation effects such that the switching rate $\lambda$ is not notably enhanced any more with an increase of $\mathrm{HU}$ concentration. We interpret this as a form of saturation from HU dosage level against the overall DNA replication stress strength, and we introduce this saturation effect into our quantitative model.

To further study the key factors controlling the activation process of the S-phase checkpoint, especially the key effector kinase Rad53, we construct various rad53 
autophosphorylation site mutants, including rad53-S350A, rad53-T354A, and rad53-S350A-T354A (called rad53SATA) (see Appendix A for the genotype), all of which alter the catalytic activity of Rad53 [48]. All rad53 mutants used had a $\operatorname{sml} 1 \Delta$ background, and $\operatorname{sml} 1 \Delta$ strain is the negative control group in Fig. 3(c). By controlling the autophosphorylation intensity of kinase $\operatorname{Rad} 53$, these rad53 mutants should cause various degrees of weakness or loss in the ASPC function. We then perform fluorescence timing tracking and analysis for the rad53 mutants under different concentrations of HU. We observe bimodal MFGR distribution (see Secs. 4.3-4.6 of the Supplemental Material [45]) and a linear inactivation curve [Figs. 3(c)-3(f)] similar to what we observed with the $\operatorname{sml} 1 \Delta$ strain, suggesting the same off-to-on switching mechanism of S-phase checkpoint activation in the rad53 mutants. The switching rates in the rad53 mutants are smaller than those seen with the $s m l 1 \Delta$ strain under the same HU stress, and the switching rate in rad53-T354A [Fig. 3(e)] is smaller still than that in rad53-S350A [Fig. 3(d)] while the rad53-SATA mutant [Fig. 3(f)] has the lowest switching rate and weakest response to DNA replication stress. In kinase Rad53, these results suggest that the T354 site plays a more important role than the S350 site and that the T354 site cooperates with the S350 site in the ASPC of yeast.

\section{BOTH DNA REPLICATION STRESS AND RAD53 AUTOPHOSPHORYLATION DETERMINE OFF-TO-ON STATE TRANSITIONS}

To reveal the dynamic mechanism underlying the activation process in the S-phase checkpoint, we built a simplified physical model involving the DNA replication stress triggered by HU stress and the autophosphorylation of Rad53 [Table I and Fig. 4(a)]. Experimental works $[49,50]$ have postulated that the cell signal transduction and amplification effects of Rad53 play important roles for controlling and transmitting diverse downstream responses to DNA replication stress. In the dimensionless form of our model, we have two crucial parameters, $p$ and $q$, which represent the DNA replication stress and intensity of Rad53 autophosphorylation, respectively [see Fig. 4(a) and Table I for their exact meanings].

Our model is based on two key assumptions. First, parameter $p$, which is related to the DNA replication stress, is an increasing function of $\mathrm{HU}$ concentration with an upper boundary. For the sake of simplicity, we assume that $p$ takes the Hill function form:

$$
p=\frac{p_{0}[\mathrm{HU}]^{n}}{K_{\mathrm{HU}}^{n}+[\mathrm{HU}]^{n}} .
$$

This assumption accounts for the saturation of the off-to-on switch rate with increasing HU level (Fig. 3 and Fig. S62 in Supplemental Material [45]), where the increase of the switch rate will saturate when the doses of HU exceed
$30 \mathrm{mM}$ in experiment. Second, Rad53 autophosphorylation parameter $q$, which is related to the cellular activation response, depends nonlinearly on the concentration of Rad53p molecules in order to realize signal amplification. We set this term in quadratic form as $q y^{2}$, which can be regarded as a simplified model for a kinetic phosphorylationdephosphorylation cycle with positive feedback [2,51,52].

The proposed deterministic model can explain the existence of off and on states. Because of the nonlinearity of the positive feedback in Rad53 autophosphorylation, the deterministic model exhibits saddle-node bifurcation behavior [Fig. 5(b)]. Within a certain range of parameter space of $p$ and $q$, two stable points exist with a low $\operatorname{Rad} 53 \mathrm{p}$ level and a high Rad53p level that correspond to the off and on states of checkpoint activation, respectively. Both DNA replication stress and Rad53 autophosphorylation are essential in the formation of two responsive states. Suppose that all cells start from the off state with a lower Rad53p level (small $y$ value in the dimensionless ODE in Table I). When the DNA replication stress increases and exceeds the threshold, the Rad53p level in all cells will be activated sharply to a high level, triggering ASPC in these cells. The required signal threshold for off-to-on state transition decreases with the increase of the strength of Rad53 autophosphorylation. However, the deterministic model fails to incorporate stochastic noise in the system; therefore, it cannot explain the cell-to-cell variability and binary response, as observed in Figs. 2 and 3.

To explain the dynamic off-to-on state switching in the single-cell experiment, we then built the stochastic model of the S-phase checkpoint activation (Table II). Here, the presence of stochasticity allows the transition among different stable states, which may be induced by the fluctuation of key kinase Rad53p, the total number of Rad53 molecules, and DNA replication stress triggered by HU stress, as well as other environmental fluctuations. A simplified model was established based on the chemical master equation (CME), which only takes the intrinsic noise of Rad53 and Rad53p into account in a single cell (Appendix B). In Fig. 4(b), we compute the switching rate $\lambda$ of the simplified model from off to on states of ASPC and fit the theoretical results with the experimental switching rates estimated from the linear region of survival curves; then, we obtain a set of acceptable ranges of parameters (see Sec. I of the Supplemental Material [45]). The extrinsic noise, mainly the fluctuation of total number $N$ of $\operatorname{Rad} 53$ in different single cells, is also discussed in Appendix C.

In order to validate the proposed model, we analyze the single-cell experiments involving $s m l 1 \Delta$, rad53-S350A, rad53-T354A, and rad53-SATA mutants, corresponding to different $\operatorname{Rad} 53$ autophosphorylation strength and different $q$ values. Similar to WT cells, the off-to-on state switching phenomenon of S-phase checkpoint activation is also observed in these mutant strains [Fig. 4(c)]. In the new fitting parameters, only the Rad53 autophosphorylation 
(a)
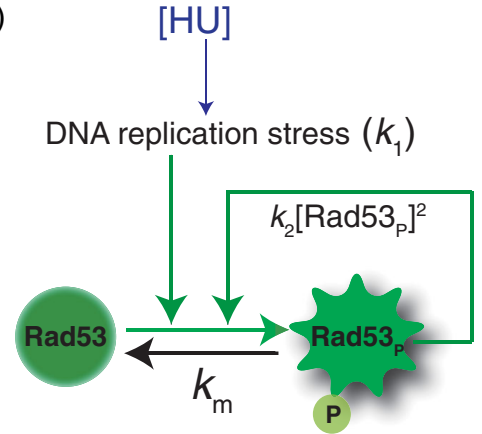

$p=k_{1} / k_{m}=p_{0}[\mathrm{HU}]^{n} /\left(K_{\mathrm{HU}}{ }^{n}+[\mathrm{HU}]^{n}\right)$

$q=k_{2}\left[\operatorname{Rad} 53_{\mathrm{Tot}}\right]^{2} / k_{\mathrm{m}}$ (b)
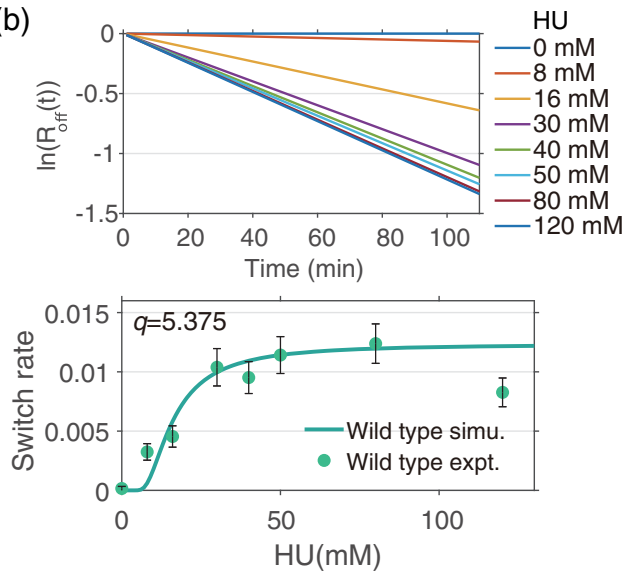

(c)
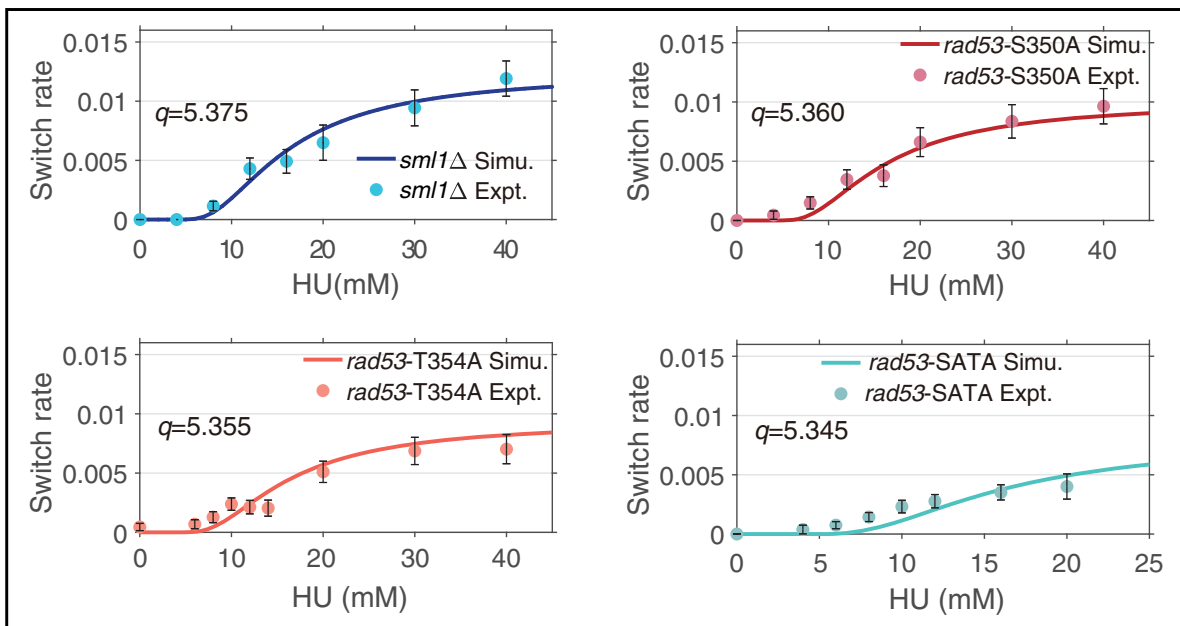

FIG. 4. Quantitative modeling for the activation process of the S-phase checkpoint (ASPC) in budding yeast and switching rate from off to on states induced by HU stress. (a) Schematic demonstration of the mathematical model. The model incorporates two dimensionless parameters, $p$ and $q$, where $p$ represents the effect of DNA replication stress induced by HU concentration, and $q$ depicts the intensity of autophosphorylation of Rad53 molecules (Table I). (b) Top panel: Quantitative results of the exponentially distributed off-to-on switching time produced by the stochastic model. Bottom panel: Switching rates from off to on states of ASPC calculated by the stochastic model for different HU concentrations (relevant to parameter $p$ ) in WT strains, which agree very well with the experimentally measured switching rates. (c) Switching rates for different Rad53 mutation strains (relevant to parameter $q$ ). The error bars denote the standard deviation of the experimental switching rates (see Sec. II of the Supplemental Material [45]). The fitted parameters in the model are $k_{m}=10^{4} \mathrm{~min}^{-1}, N=1000, K_{\mathrm{HU}}=2 \mathrm{mM}, n=2, p_{0}=4.38 \times 10^{-2}, q_{\mathrm{WT}}=5.375, q_{\text {sml1 }}=5.375$, $q_{\mathrm{SA}}=5.360, q_{T A}=5.355$, and $q_{\mathrm{SATA}}=5.345$.

intensity parameter $q$ in the mutant strain model $(q=5.360$ in $\mathrm{rad} 53-\mathrm{S} 350 \mathrm{~A}, q=5.355$ in $\mathrm{rad} 53-\mathrm{T} 354 \mathrm{~A}, q=5.345$ in rad53-SATA) differed from the setting of the WT and $\operatorname{sml1\Delta }$ strain models $(q=5.375$ in both strain models). These results suggest that the mutation in S350A and T354A can reduce $\operatorname{Rad} 53$ autophosphorylation intensity, leading to the decrease of off-to-on switching rates under the same HUinducing signal. According to the theoretical analysis, we further confirmed that T354 may be a more essential phosphorylation site in the Rad53 activation pathway compared to S350.

These results demonstrate that the DNA replication stress signal transduction and amplification effects of Rad53 in our theoretical model capture the essence of S-phase checkpoint activation.

\section{STOCHASTIC NOISE DRIVES BARRIER-CROSSING SWITCHING IN CHECKPOINT ACTIVATION}

We have explored the underlying biological mechanisms with the combination of single-cell experiments and theoretical modeling. Now, we try to understand the off-to-on switching in S-phase checkpoint activation from the dynamic perspective. Specifically, we focus on explaining how the stress signal and amplification mechanism would result in the switching between two responsive states.

As revealed by the theoretical modeling, the initial driving force of the switch turns out to be the random noise in the biochemical process. At the beginning of the $\mathrm{S}$ phase, most Rad53 molecules in most yeast cells are not 
TABLE I. Deterministic model of Rad53 phosphorylation.

\begin{tabular}{|c|}
\hline $\begin{array}{c}\text { ODE dynamics } \\
d x / d t=R^{+}(x)-R^{-}(x)=\left(k_{1}+k_{2} x^{2}\right)(R-x)-k_{m} x\end{array}$ \\
\hline $\begin{array}{l}\text { Variables and parameters } \\
x: \text { Concentration of phosphorylated Rad53 (Rad53p) } \\
R: \text { Total concentration of Rad53 molecules } \\
k_{1}: \text { Rad53 phosphorylation rate induced by DNA replication } \\
\text { stress signal } \\
k_{2}: \text { Rad53 autophosphorylation rate } \\
k_{m}: \text { Rad53 dephosphorylation rate }\end{array}$ \\
\hline $\begin{array}{c}\text { Dimensionless ODE } \\
d y / d \tau=\left(p+q y^{2}\right)(1-y)-y\end{array}$ \\
\hline $\begin{array}{l}\text { Variables and parameters } \\
y=x / R: \text { Proportion of Rad53p } \\
p=k_{1} / k_{m}=p_{0}[\mathrm{HU}]^{n} /\left(K_{\mathrm{HU}}^{n}+[\mathrm{HU}]^{n}\right): \text { Intensity of the DNA } \\
\text { replication stress }(\mathrm{HU}) \\
q=k_{2} R^{2} / k_{m}: \text { Intensity of Rad53 autophosphorylation } \\
\tau=k_{m} t \text { : Rescaled time }\end{array}$ \\
\hline
\end{tabular}

TABLE II. Stochastic model of Rad53 phosphorylation.

\begin{tabular}{|c|}
\hline $\begin{array}{c}\text { Chemical master equation } \\
\partial_{t} p_{n}(t)=u_{n-1} p_{n-1}(t)+w_{n+1} p_{n+1}(t)-\left(u_{n}+w_{n}\right) p_{n}(t)\end{array}$ \\
\hline $\begin{array}{l}\text { Variables and parameters } \\
p_{n}(t) \text { : Probability of finding } n \operatorname{Rad} 53 \mathrm{p} \text { molecules at time } t \\
N=R V \text { : Total number of } \operatorname{Rad} 53 \text { and } \operatorname{Rad} 53 \mathrm{p} \text { molecules } \\
\text { in a yeast cell } \\
V \text { : System size of the chemical reactions (a yeast cell) } \\
u_{n}=\left[k_{1}+\left(k_{2} / V^{2}\right) n^{2}\right](N-n)=k_{m}\left(p+q n^{2} / N^{2}\right)(N-n) \\
w_{n}=k_{m} n\end{array}$ \\
\hline
\end{tabular}

phosphorylated; therefore, these cells are in the off state with a low Rad53p level. As time progresses, however, noise brings fluctuations to the Rad53p level, and in some cells, the deviation from stable states will be amplified owing to the autophosphorylation mechanism, which finally drives the cells to the on state where most effector kinases become phosphorylated. An intermediate state, or saddle point in the deterministic model, exists between the off and on states, balancing between the attraction force to the off state and the autophosphorylation effect that drags the cell towards the on state. The molecular switching process is determined by the noise according to the number of $\operatorname{Rad} 53$ molecules $\left(N \sim 10^{3}\right.$ in each cell) [53] and fast microscopic phosphorylation and dephosphorylation reaction timescales $\left(k_{1} \sim 10^{2} \mathrm{~min}^{-1}, k_{m} \sim 10^{4} \mathrm{~min}^{-1}\right)$ [54].

The quasipotential landscape perspective [55-59] based on stochastic models provides a vivid description of this stochastic off-to-on switching of S-phase checkpoint activation shown in Fig. 5(a). The quasipotential landscape of the system $\varphi(y)$ can be constructed as the zero noise limit of steady-state distribution (Appendix C), where the macroscopic state $y$ is the proportion of phosphorylated Rad53 molecules within the cell. The two local minima of the double-well quasipotential correspond to the two stable points in the deterministic model and, therefore, the off and on states of S-phase checkpoint activation, respectively. Hence, off-to-on state switching can be viewed as the transition from one metastable state to another. With stochastic noise, the system or the cell will fluctuate around the off state, cross over a potential barrier, and finally reach the on state. The quasipotential barrier height is determined by the parameters $p$ and $q$, reflecting the difficulty of switching from the off to on state. In fact, the switching rate $\lambda \propto \exp (-\Delta \varphi)$ [60], where the barrier height $\Delta \varphi=\varphi\left(y_{\text {saddle }}\right)-\varphi\left(y_{\text {off }}\right)$.

The dependence of barrier heights on parameters $p$ and $q$ is calculated and shown in Fig. 5(b). We find that the increase of DNA replication stress reduces the barrier height and, thus, facilitates the off-to-on switching, while the decrease of the Rad53 autophosphorylation strength increases the barrier height and makes off-to-on switching more difficult to achieve.

\section{FURTHER THEORETICAL IMPLICATIONS AND EXPERIMENTAL VERIFICATION}

According to our theoretical results [Fig. 5(b)], if we further decrease Rad53 autophosphorylation intensity $q$ below the rad53-SATA level, a threshold phenomenon for different HU stimulations will appear. If the $q$-valued mutant strain still lies in the bistable region of the deterministic model, while the switching rate can be ignored for small $p$ values (for instance, $\lambda<0.01$ per cell cycle such that offto-on switching only occurs with rare probability less than $1 \%$ ), then the binary activation response will only be observed for HU stimulation greater than a threshold level. By contrast, if the $q$ value of the mutant strain falls into the monostable parameter space, then the graded response might apply. To verify this prediction, we construct the dimeric Rad53 binding surface mutant, rad53-K231E-R232DK233E (named rad53-KRK-EDE), to make an extreme reduction of the autophosphorylation intensity. The KRKEDE mutant that changes the Rad53 dimer binding sites, making it difficult to form the Rad53 dimer, may fail to activate the S-phase checkpoint pathway, which results in the stagnation of the replication fork, cell growth arrest, and even apoptosis under high replication stress.

The experimental evidence strongly supports our theoretical implications. In Figs. 5(c) and 5(d), we find that the bimodal distributions disappear under both 1-mM and 12$\mathrm{mM} \mathrm{HU}$ concentrations, irrespective of data processing by tSNE or our analytic approach via MFGR. The internal mechanism of this phenomenon can be explained through the quasipotential landscape perspective, as shown in Fig. 5(a). Since mutations at these sites severely affect the autophosphorylation intensity of Rad53, the S-phase checkpoint pathway can only be partially activated, and the replication 
(a)

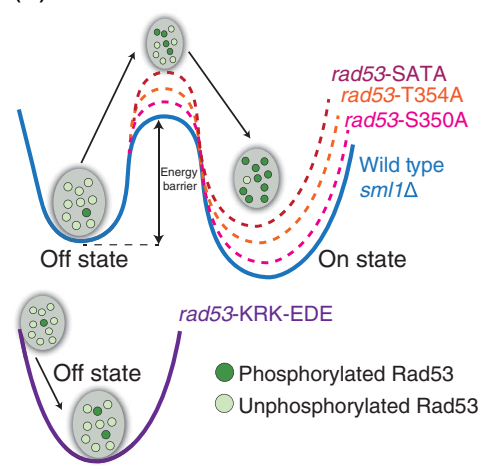

(d) (b)

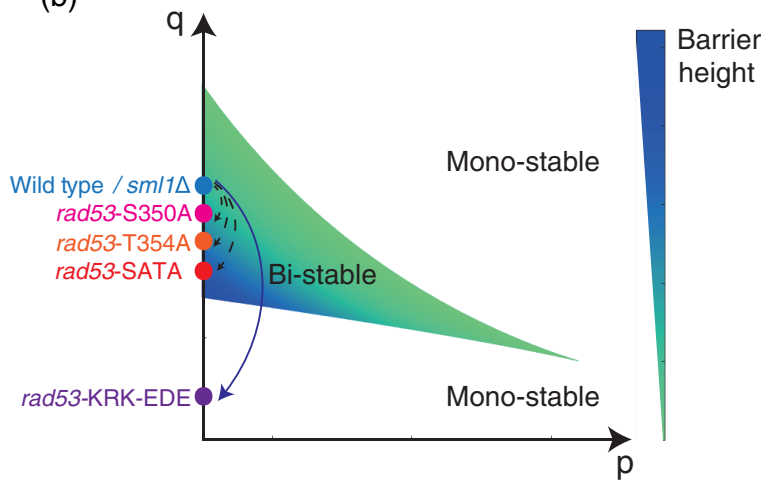

(c)

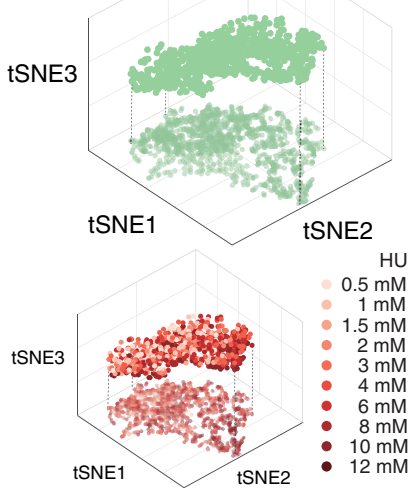

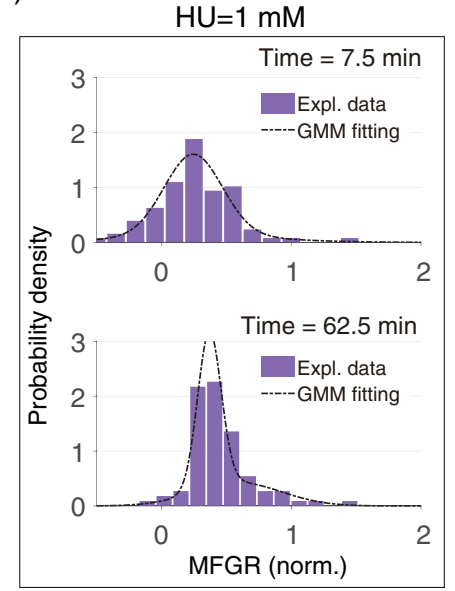

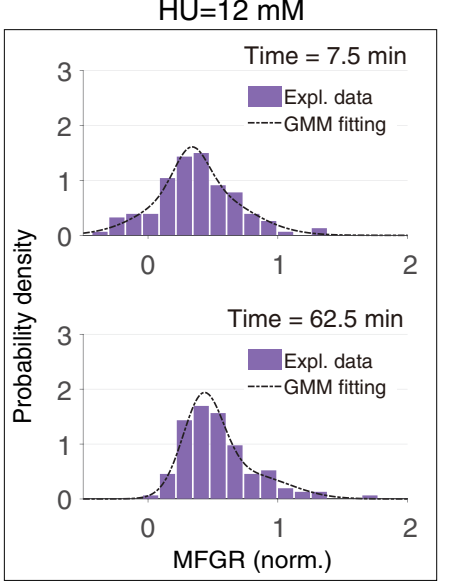

(e)

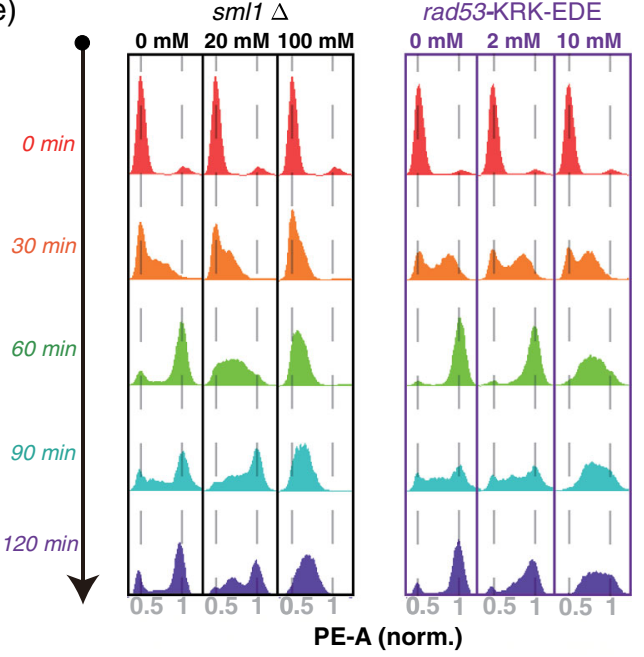

FIG. 5. Quasipotential perspective for bimodal and unimodal distribution in rad53 mutants. (a) Quasipotential landscape perspective for the activation process of the yeast S-phase checkpoint. The off and on states correspond to the two potential wells in the quasipotential landscape, and the activations need to cross the potential barrier between the off state and the saddle state. The dimeric Rad53 binding the surface mutant rad53-KRK-EDE demonstrates the potential landscape without a potential barrier, which displays a unimodal distribution of MFGR. (b) Dependence of barrier heights in the off-to-on switches on the parameters $p$ (signal intensity) and $q$ (Rad53 autophosphorylation intensity) in the stochastic model. The increase of HU concentration will lower the barrier height to enable switching and facilitate the transition, while the suppression of autophosphorylation will increase the potential barrier and impede the transition. The corresponding $q$ values of various mutant strains are marked on the map. (c) Raw data of a time series with various HU doses, processed by the t-SNE dimensionality reduction method. (d) Unimodal distribution of normalized MFGR of the rad53-KRK-EDE mutant for $1-\mathrm{mM}$ and $10-\mathrm{mM}$ HU every $5 \mathrm{~min}$. (e) Flow chart of rad53-KRK-EDE strain and $s m l 1 \Delta$ strain cultured at different concentrations of HU. All cells are synchronized in the G1 phase by a $\alpha$ factor at 0 min. It can be observed that the rad53-KRK-EDE mutant is arrested in the $\mathrm{S}$ phase under $\mathrm{HU}=10 \mathrm{mM}$, suggesting its relatively poor tolerance to high $\mathrm{HU}$ doses.

fork is stalled when these mutants are affected by HU beyond their tolerance. We further use flow cytometry to analyze the cell cycles at different HU concentrations. The result shows that budding yeast cannot accomplish DNA replication when submitted to high concentrations of HU [Fig. 5(e)], e.g., the DNA content of the rad53-KRK-EDE mutant is between one copy (G1 phase) and two copies (G2 phase) under $10 \mathrm{mM}$ HU. For low HU concentrations, compared with the untreated strain, some of the cells can complete DNA replication and evolve from the $S$ phase to the G2 phase, while the others are arrested in the $S$ phase. Different mutants have divergent autophosphorylation intensities, so they have different tolerances to $\mathrm{HU}$ treatments. The strains with weaker autophosphorylation intensity will be arrested in the $\mathrm{S}$ phase under lower concentrations of HU; however, the WT strain with intact autophosphorylation requires about $100 \mathrm{mM} H \mathrm{HU}$ before arrest in the S phase.

In addition to validating the unimodal distribution of MFGR for the small $q$-valued mutant strains, our theoretical model can also provide meaningful insights about the activation mechanisms of the S-phase checkpoint beyond the activation stage. For example, we can make reasonable inferences about Rad53-overexpression strains. In such strains, the gene regulating the synthesis of $\operatorname{Rad} 53$ will 
be overexpressed; in other words, we increase the parameter $R$ in the model. Since $q=k_{2} R^{2} / k_{m}$ and $N=R V$, the parameters $q$ and $N$ are both increased. If the overexpression rate of Rad53 is more than 1.7-fold beyond that of WT strain, our simulation results suggest that the off-to-on switching rate $\lambda$ will increase to the saturation level $\left(\lambda=0.03 \mathrm{~min}^{-1}\right)$ and that the S-phase checkpoint will be activated, even with very low HU concentration. We leave further experimental verification as a future task.

\section{CONCLUSION}

Despite abundant knowledge about the S-phase checkpoint, few quantitative studies have reported on the in vivo dynamics of checkpoint activation at the single-cell level. In this paper, we studied the activation process of the Sphase checkpoint in budding yeast, which is triggered by HU stress. We developed the single-cell experiment and data analysis method and constructed the quantitative dynamic models to reveal the role of noise in signal transduction pathways of living cells. Our minimal model embraces the significance of stochastic noise to conduct the appearance of enough population of the key kinase Rad53p, which is the basis for the subsequent amplification induced by autophosphorylation. From the experiments on rad53-S350A, rad53-T354A, and rad53-SATA mutants, we directly verified that the reduction of autophosphorylation intensity leads to a decreased off-to-on switching rate and that the rad53-KRK-EDE mutant shows a unimodal distribution of MFGR.

In our work, we focused on the activation process of the S-phase checkpoint triggered by different HU concentration levels. We introduced and defined maximum values of FGR for the Rnr3 protein, MFGR $(t)$, to depict whether a yeast cell has been triggered to the on state of the S-phase checkpoint in the interval $[0, t]$, together with the inactivation curve $R_{\text {off }}(t)$ to represent the proportion of cells that have not been activated and remain in the off state in $[0, t]$. Then, the time evolution curve $R_{\text {off }}(t)$, as shown in Figs. 3(b)-3(f), describes the first off-to-on state transition under various $\mathrm{HU}$ doses. We note that although MFGR could be a good indicator to describe the activation process of the S-phase checkpoint, the downstream feedback of the repair pathway can result in more complex dynamics, including the subsequent decrease and even oscillation of FGR after activation (see Sec. III. 6 in the Supplemental Material [45]), which is also the focus of our future work.

Here, we analyzed and derived the first passage time distribution $R_{\text {off }}(t)$ in the quantitative model as well as the state-transition rate of the stochastic process [61] to depict the dynamics of S-phase checkpoint activation. Our results also suggest that the increase of HU doses in higher levels induces the saturation of the increase of switching rates. Combining single-cell experiments and theoretical modeling, we conclude that the S-phase checkpoint activation of the budding yeast cell cycle is a stochastic switching process from off to on states triggered by noise. This result suggests the existence of a binary response instead of a graded response in the dynamical process, which has been previously explored in the context of gene expression patterns [15]. In addition to the intrinsic chemical-reaction noise considered in the main text, we also analyzed the effects of extrinsic noise resulting from molecular number fluctuations (Appendix C 4) and growth heterogeneity [62] (Appendix C5), whose effects could also be further explored in the downstream process after the activation of the S-phase checkpoint.

While stochastic noise provides the initial thrust to trigger such switching, the nonlinear autophosphorylation of Rad53 plays the major role in amplifying HU-induced DNA replication stress and producing the off-to-on switching mechanism. We also inspected an alternative explanation of nonlinearity in the Rnr complex by HU inhibition, which might be inconsistent with our experimental findings (Appendix C 6).

We adopted the quasipotential landscape perspective to understand S-phase checkpoint activation as a barriercrossing process. In physics, an important theoretical implication about the noise-induced transitions for dynamics confined in potential wells or attractor basins is the exponentially distributed dwell time $[63,64]$. Our experiment and analysis contributed to the evidence of its existence on the single-cell trajectory level in the S-phase checkpoint activation process. Among different proposals for constructing a quasipotential landscape in biochemical systems $[51,52,57,59]$, we chose to calculate the quasipotential barrier to quantify the asymptotic off-to-on switching rate, which is inversely proportional to the mean first exit time from the off domain. We remark that this approach is still valid, even when the considered switching is a nonequilibrium process and the downstream reaction schemes are changed after the transition.

More generally, in a certain population of cells, the role of noise, or stochasticity, in cell signal transduction pathways arises from both random fluctuations in cellular chemical reactions and the different distinct states of the cells. If hundreds of mammalian cells are involved, they should be fully differentiated, and there should be a large variability of cell states, including different genotypes, phenotypes, and various cell functions. More importantly, a mammalian cell is much larger than a yeast cell in volume and has more molecules than a yeast cell. For example, a HeLa cell is about $2000 \mu \mathrm{m}^{3}$ in volume with 160000 p53 molecules per cell [65], while a budding yeast cell is $50 \mu \mathrm{m}^{3}$ in volume with 1000-2000 Rad53 molecules [53]. In these mammalian cells, the variability of cell states may be a dominant source of cellular stochasticity, promoting the engagement of the graded response. However, in a population of budding yeast cells cultured overnight to form a single yeast clone, they share the same genotype. The random fluctuations of chemical reactions inside the 
yeast cells are easier observe. In this work, we observed the off-to-on state transition in the S-phase checkpoint activation with a positive feedback loop. Therefore, our results suggest that stochasticity in this signal transduction pathway is mainly caused by the cellular chemical reaction, which is very consistent with the stochastic model. Similar noise-induced transition models have been proposed to explain and understand biological processes, such as the enhancement of cellular memory [61] and the competence regulation in Bacillus subtilis $[66,67]$. We have learned that cells not only utilize special biological circuits to make reliable decisions in the noisy environment [17] but also take advantage of noise [68] in some biological processes.

Furthermore, our results and analysis suggest that singlecell microfluidic measurements may provide a new approach to study key kinase protein activity dynamically in the cell signal transduction pathway. The quantitative framework presented in this paper-such as the inference techniques about phosphorylation-level dynamics, the survival analysis perspectives, and the quasipotential landscape point of view-can also be utilized to investigate similar biological processes.

\section{ACKNOWLEDGMENTS}

The authors are grateful to Yongqiang Jia, Xiaodong Yan, Xiao Li, Dr. Jianfeng Pei, Dr. Xiaojing Yang, Dr. Hong Qian, and Dr. Chao Tang for helpful discussions. This work was supported by the National Key R\&D Program in China (Grants No. 2018YFA0900200 and No. 2020YFA0906900) and the National Natural Science Foundation of China (Grants No. 11421101, No. 11825102, and No. 12090051). T. L. was also partially supported by Beijing Academy of Artificial Intelligence (BAAI). The authors thank the anonymous reviewers for their insightful comments and helpful suggestions to improve the manuscript.

\section{APPENDIX A: MATERIALS AND METHODS IN EXPERIMENTS}

In this Appendix, we introduce the materials and methods in conducting the single-cell experiment.

\section{Yeast strains}

We studied the activation process of the S-phase checkpoint in budding yeast Saccharomyces cerevisiae BY4741. To quantitatively analyze the activation level of kinase Rad53 (especially phosphorylated Rad53), we fused the GFP to the C-terminal of Rnr3 as the reporter protein (noted as BY4741 RNR3-GFP, WT), where Rnr3 is the downstream protein of the Rad53 transduction pathway that is transcribed and translated when the S-phase checkpoint is activated. Thus, in a single cell, the growth rate of Rnr3 with time reflects the level of phosphorylated Rad53 $(\operatorname{Rad} 53 p)$.

In order to construct several rad53 mutant strains, we knocked out the SML1 gene in BY4741 RNR3-GFP (noted as BY4741 sml1s RNR3-GFP); otherwise, rad53 mutant strains cannot survive in the construction process. Based on the BY4741 sml1 $R$ RNR3-GFP strain, we constructed several rad53 mutant strains: S350 to alanine (noted as rad53-S350A), T354 to alanine (noted as rad53-T354A), both S350 and T354 to alanine (noted as rad53-S350A-T354A, and also as rad53-SATA), and a triple mutation (noted as rad53-K231E-R232D-K233E, and also as rad53-KRK-EDE, in which K231 to glutamic acid, R232 to aspartic acid, and K233 to glutamic acid). The genotypes of all strains used in this study are summarized in Table III.

\section{Cell culture}

Yeast cells were regularly cultured in YPAD solid medium and stored at $4{ }^{\circ} \mathrm{C}$. The procedure of cell culture before imaging is as follows. First, we picked a single

TABLE III. Yeast strains used in this study.

\begin{tabular}{|c|c|c|}
\hline Strains & Genotype & Source \\
\hline BY4741 & MATa his $3 \Delta 1$ leu $2 \Delta 0$ met $15 \Delta 0$ ura $3 \Delta 0$ lys $2 \Delta 0$ & In stock \\
\hline BY4741 RNR3-GFP & MATa his $3 \Delta 1$ leu $2 \Delta 0$ met $15 \Delta 0$ ura $3 \Delta 0$ lys $2 \Delta 0$ RNR3-GFP::HIS3 & This study \\
\hline BY4741 sml1 $\Delta$ & $\begin{array}{l}\text { MATa his } 3 \Delta 1 \text { leu } 2 \Delta 0 \text { met } 15 \Delta 0 \text { ura } 3 \Delta 0 \text { lys } 2 \Delta 0 \\
\quad \text { RNR3-GFP:: HIS } 3 \operatorname{sml} 1 \Delta:: \text { NatMX }\end{array}$ & This study \\
\hline $\operatorname{rad} 53-S 350 A$ & $\begin{array}{l}\text { MATa his } 3 \Delta 1 \text { leu } 2 \Delta 0 \text { met } 15 \Delta 0 \text { ura3 } \Delta 0 \text { lys } 2 \Delta 0 \\
\quad \text { RNR3-GFP::HIS3sml1 : :NatMX rad53-S350A::LEU2 }\end{array}$ & This study \\
\hline $\operatorname{rad} 53-T 354 A$ & $\begin{array}{l}\text { MATa his } 3 \Delta 1 \text { leu } 2 \Delta 0 \text { met } 15 \Delta 0 \text { ura } 3 \Delta 0 \text { lys } 2 \Delta 0 \\
\quad \text { RNR3-GFP::HIS3sml } 1 \Delta:: \text { NatMX rad53-T354A: :LEU2 }\end{array}$ & This study \\
\hline rad53-S350A-T354A & $\begin{array}{l}\text { MATa his } 3 \Delta 1 \text { leu } 2 \Delta 0 \text { met } 15 \Delta 0 \text { ura } 3 \Delta 0 \text { lys } 2 \Delta 0 \\
\quad \text { RNR3-GFP::HIS3sml } 1 \Delta:: \text { NatMX rad53-S350A-T354A::LEU2 }\end{array}$ & This study \\
\hline $\begin{array}{l}\operatorname{rad} 53-K 231 E-R 232 D- \\
\quad K 233 E\end{array}$ & $\begin{array}{l}\text { MATa his } 3 \Delta 1 \text { leu } 2 \Delta 0 \text { met } 15 \Delta 0 \text { ura } 3 \Delta 0 \text { lys } 2 \Delta 0 \\
\quad \text { RNR3-GFP::HIS3mml1 }:: \text { NatMX rad53-K231E-R232D-K233E::LEU2 }\end{array}$ & This study \\
\hline
\end{tabular}


colony and cultured it overnight in $5 \mathrm{ml}$ YPAD liquid medium at $30^{\circ} \mathrm{C}$. The OD600 of culture in YPAD will reach $0.8-1$ within $8-10$ hours, which ensures that the cells are in the logarithmic phase and have optimal growth conditions. After overnight incubation in YPAD, we harvested the cells and resuspended them in transparent synthetic complete (SC) liquid medium to avoid background fluorescent noise induced by yellow YPAD liquid medium. Then, the cells (initial OD600 $=0.1-0.2$ ) grew in the SC liquid medium to an OD600 of 0.4-0.6 after 4-5 hours at $30^{\circ} \mathrm{C}$. Next, we harvested the cells and resuspended them in 50- $\mu$ l fresh SC medium to increase the cell density. Finally, we injected the cells into the chamber and cultured the cells for 2 hours to ensure that they were able to adapt to the new environment before imaging.

\section{Media and chemicals}

All of the media in experiments and their components are listed below.

(i) YPAD medium contains $2 \%(\mathrm{w} / \mathrm{v})$ glucose, $20 \mathrm{~g} / \mathrm{L}$ peptone, $10 \mathrm{~g} / \mathrm{L}$ yeast extract, and $120 \mathrm{mg} / \mathrm{L}$ adenine.

(ii) $\mathrm{SC}$ medium contains $2 \%(\mathrm{w} / \mathrm{v})$ glucose, $1 \mathrm{X}$ amino acid drop-out supplements, $6.7 \mathrm{~g} / \mathrm{L}$ yeast nitrogen base without amino acids, $100 \mathrm{mg} / \mathrm{L}$ leucine, $20 \mathrm{mg} / \mathrm{L}$ histidine, $20 \mathrm{mg} / \mathrm{L}$ tryptophan, $20 \mathrm{mg} / \mathrm{L}$ adenine, and $20 \mathrm{mg} / \mathrm{L}$ urea. The formula of $1 \mathrm{X}$ amino acid drop-out supplements is $20 \mathrm{mg} / \mathrm{L}$ arginine, $20 \mathrm{mg} / \mathrm{L}$ methionine, $30 \mathrm{mg} / \mathrm{L}$ tyrosine, $30 \mathrm{mg} / \mathrm{L}$ isoleucine, $30 \mathrm{mg} / \mathrm{L}$ lysine, $50 \mathrm{mg} / \mathrm{L}$ phenylalanine, $100 \mathrm{mg} / \mathrm{L}$ glutamic acid, $100 \mathrm{mg} / \mathrm{L}$ aspartic acid, $150 \mathrm{mg} / \mathrm{L}$ valine, $200 \mathrm{mg} / \mathrm{L}$ threonine, and $400 \mathrm{mg} / \mathrm{L}$ serine.

(iii) $\mathrm{SC}$ medium with different concentrations of $\mathrm{HU}$ included a mixture of SC liquid medium and $1 \mathrm{M}$ HU stock solution.

(iv) Glucose was purchased from Ameresco, Solon, $\mathrm{OH}$. Peptone was purchased from Becton, Dickinson and Company, Sparks, MD. All other reagents were purchased from Sigma-Aldrich, St. Louis, MO.

\section{Microfluidic chip}

The microfluidic chip was designed with a slight modification from Luo et al. [69] (shown in Fig. 6), which is followed by a standard soft lithography process to fabricate the PDMS poly (polydimethylsiloxane). L-edit software was used to design the microfluidic pattern. Each chip contains four channels that can be independently loaded, and each channel contains 40 chambers evenly distributed on both sides of the channel. The size of each channel is $20 \mathrm{~mm} \times$ $200 \mu \mathrm{m} \times 10 \mu \mathrm{m}$ (length $\times$ width $\times$ height), and the size of each chamber is $200 \mu \mathrm{m} \times 200 \mu \mathrm{m}$ (length $\times$ width), which ensures that cells will not overlap and be extruded by the chamber. The height of the chamber is designed to be slightly higher than the diameter of the budding yeast cell on average (around $4 \mu \mathrm{m}$ ). We use a TS-1B injection pump produced by

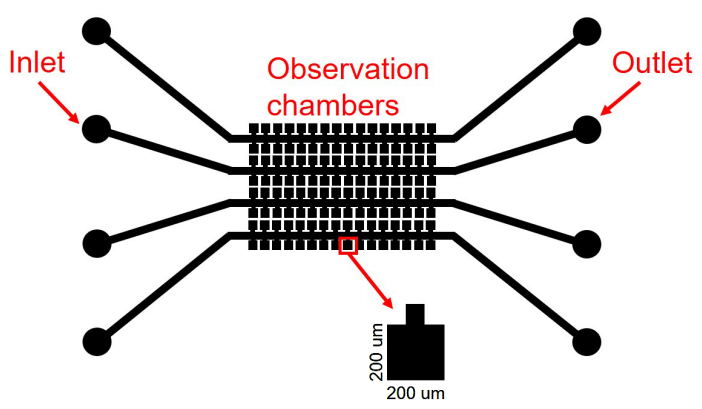

FIG. 6. Schematic diagram of our independent four-channel microfluidic chip. The image shows the structure of the channel, which consists of the main sulcus and the lower chambers on both sides. These chambers are designed for cell observation. Some of the cells are forced into the chamber after the bacteria is injected through the inlet. The culture is then passed through the inlet to ensure that the cells can grow normally. All specific parameters of our chip are shown in Appendix A 4.

Longer pump company accompanied by a 1-ml injector to inject a SC or HU solution into the chip. The speed of the injection pump is $66.67 \mu \mathrm{l} / \mathrm{hr}$.

\section{Time-lapse microscopy}

The procedure of our single cell imaging experiment is as follows.

(i) Inoculate $5 \mathrm{ml}$ of YPAD with a single colony of yeast strain to make a preculture. Incubate at $30{ }^{\circ} \mathrm{C}$ with stirring until the OD600 of the culture is 0.8-1.0.

(ii) Transfer the yeast cells from YPAD to $\mathrm{SC}$ to have OD600 of about $0.1-0.2$ and incubate at $30^{\circ} \mathrm{C}$ with stirring until the OD is $0.4-0.6$.

(iii) Harvest the cells by centrifugation at $2500 \mathrm{rpm}$ in a clinical centrifuge (Eppendorf Centrifuge 5417R) for 2 minutes. Decant the supernatant, and then resuspend cells in the remaining medium to increase the concentration of the cell solution. Pump the cells into the microfluidic chip with an injection syringe in a moderate force to obtain an appropriate cell density in each chamber.

(iv) Observe cells with a time-lapse microscope. In the first step, we feed cells with SC to ensure the cells adapt to the external environment for 2 hours while observing the cell growth status with the bright field (bf) channel. In the next step, we switch to the SC medium containing $\mathrm{HU}$ to apply replication pressure to the cells and observe the fluorescence of Rnr3 during the first cycle.

(v) 5 . We select no more than 120 observation points from the microfluidic chip using the NIS-Elements software included in the microscope system. First, cells are photographed using only the bf channel to confirm their growth status and to exclude those unsatisfactory observation points. Then, cells are 
photographed through both the bright field and the green fluorescence channel. Each observation point is shot every 5 minutes for a total of 8 hours.

We set the microfluidic flow system in a constant temperature incubator (World Precision Instruments, Sarasota, FL, USA) at $30^{\circ} \mathrm{C}$ to provide cells with an optimal environment. Time-lapse movies are obtained with epifluorescence microscopy using a Nikon Ti-E inverted microscope equipped with the objective lens Plan Apo VC 60X/1.40 Oil DIC N2, the motorized XY Stage and the Prefect-Focus System (Nikon Co., Tokyo, Japan). We image each observation point every 5 minutes with an EMCCD (Evolve 512, Photometrics, Tucson, AZ, USA) and Lambda SC shutter controllers (Sutter Instrument Co., Novato, CA, USA). NIS Elements AR v4.13 software, which comes with the system (Nikon Co., Tokyo, Japan), is used to automate image acquisition and microscope control.

\section{Image analysis}

The fluorescence acquisition algorithm of the original image sequence is the same as that of Yang et al. [70]. We utilize the MATLAB program cellseg to obtain the area of each cell and calculate the mean fluorescence intensity of each cell over the entire observation period. Then, we use the software ImageJ to calculate the background fluorescence intensity of each frame, and we revise the mean fluorescence intensity of each cell after subtracting the background fluorescence value from the original mean fluorescence value. Considering that HU mainly affects the response of cells in their $\mathrm{S}$ phase, here we regard the budding moment of yeast cells as the beginning of the $\mathrm{S}$ phase and only extract data of normal morphological cells from the first budding moment to the second budding moment or to the end of the shot. For each concentration of $\mathrm{HU}$, we calculate hundreds of cells to characterize population-level behavior.

To obtain the growth rate of Rnr3-GFP of each cell and remove the perturbation caused by noise, we use a 15 -min time-window fitting to calculate the rate of the Rnr3-GFP expression level, which moves from the start to the end of the time series. Then, we linear fit to the data points in each window and use the fitted slope at the middle instant of the window as the growth rate of Rnr3-GFP. More details about the analysis method can be found in Sec. II of the Supplemental Material [45]. The effect of the fitting time range is also investigated in Sec. III. 5 of the Supplemental Material [45].

\section{APPENDIX B: PHYSICAL MODELS}

In this Appendix, we demonstrate the basic setup of our physical models (including both deterministic and stochastic models) to explain the experimental facts.

\section{Simplified reactions in S-phase checkpoint}

As demonstrated in Figs. 1(a) and 4(a) of the main text, the phosphorylation mechanism of the effector kinase Rad53 can be viewed as the core module in the S-phase checkpoint pathway under study. Therefore, the gene regulation network of S-phase checkpoint activation can be simplified into a one-dimensional model, focusing on the transformation of Rad53 molecules between phosphorylated and unphosphorylated states (Fig. 7). Previous experimental works suggested that the phosphorylation of effector kinase in response to replication stress consists of two stages [71]: the replication-stress-induced activation of Rad53 kinase promoted by the adaptor protein Mrc1, and (a)

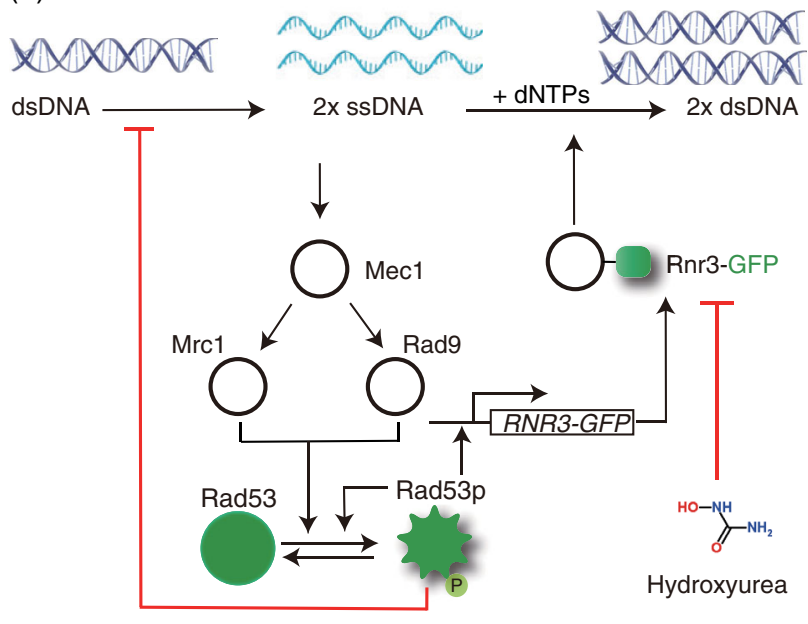

(b)

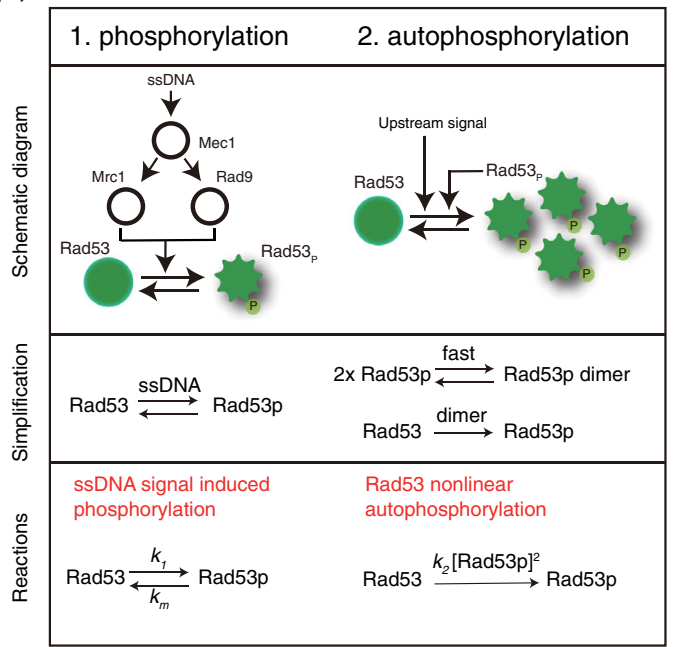

FIG. 7. (a) Schematic diagram for the chemical reactions in Rad53 phosphorylation in the S-phase checkpoint pathway. (b) Two main processes of Rad53 phosphorylation. The left column denotes the phosphorylation induced by the upstream signal, and the right column denotes the autophosphorylation of Rad53. 
the autophosphorylation of Rad53 molecules via dimerization. Correspondingly, in the simplified model, the phosphorylation reaction of Rad53 also incorporates both the signal transduction and the nonlinear amplification effects.

These assumptions lead to the deterministic and stochastic model of Rad53 phosphorylation shown in Tables I and II of the main text.

\section{Derivation of off-on switch rate}

As discussed in the main text, we are particularly interested in the switch rate from one state to another. For instance, how many cells will transit from the off state (where the Rad53p number is low) to the on state (where the Rad53p number is high) in the S-phase checkpoint per unit time? This question is closely related to the notion of mean first passage time in the theory of stochastic processes.

For the cell that initially has $i \operatorname{Rad} 53 p$ molecules, define $\tau_{i}^{n}$ as the first time point at which it has $n$ phosphorylated effector kinase, which is also known as the first passage time. Denote $\mathbb{E} \tau_{i}^{n}=T_{i}^{n}$, representing the mean of the first passage time to $n$ starting from $i$. Within the initial $\Delta t$ interval, there is probability $w_{i} \Delta t+o(\Delta t)$ to dephosphorylate one $\operatorname{Rad} 53 \mathrm{p}$, probability $u_{i} \Delta t+o(\Delta t)$ to phosphorylate another $\operatorname{Rad} 53$, and probability $1-\left(u_{i}+w_{i}\right) \Delta t+$ $o(\Delta t)$ that the Rad53p number remains. Therefore,

$$
\begin{aligned}
T_{i}^{n}= & \Delta t+w_{i} \Delta t T_{i-1}^{n}+u_{i} \Delta t T_{i+1}^{n} \\
& +\left(1-\left(u_{i}+w_{i}\right) \Delta t\right) T_{i}^{n}+o(\Delta t),
\end{aligned}
$$

which gives

$$
w_{i} T_{i-1}^{n}-\left(u_{i}+w_{i}\right) T_{i}^{n}+u_{i} T_{i+1}^{n}=-1,
$$

with the boundary condition $T_{n}^{n}=0$ (note that $w_{0}=0$, and thus only one additional condition is needed).

The analytical solution is available in compact form [72] through

$$
T_{i}^{n}=\sum_{m=i+1}^{n} \sum_{l=0}^{m+1} \frac{P_{l}^{\mathrm{st}}}{w_{m} P_{m}^{\mathrm{st}}},
$$

where $P^{\text {st }}$ is the steady-state distribution to CME,

$$
P_{k}^{\mathrm{st}}=P_{0}^{\mathrm{st}} \prod_{l=0}^{k-1} \frac{u_{l}}{w_{l+1}} .
$$

Therefore, the theoretical off-on switch rate is obtained by $\lambda=1 / T_{i^{*}}^{n^{*}}$, setting $i^{*}$ as the number of molecules corresponding to the low state, and $n^{*}$ the high state. In fact, the value of $T_{i^{*}}^{n^{*}}$ is relatively stable for the choice of $n^{*}$ within the same attractor (similar results also hold for different choices of the starting point of $i^{*}$-see Sec. III. 4 of the Supplemental Material [45] for more detailed discussions).

On the other hand, the experimental off-on transition rate $\hat{\lambda}$ under different HU doses can be estimated in the linear regime of the survival curve as shown in Fig. S6. Combining it with the analytical results obtained by a suitable choice of parameters according to experimental data (see Sec. I of the Supplemental Material [45]), we arrive at Figs. 4(c)-4(f) in the main text.

\section{APPENDIX C: MODEL ANALYSIS AND RESULTS}

In this Appendix, we discuss the results derived from our physical models and the relevant insights into the S-phase checkpoint activation mechanism.

\section{Barrier crossing and quasipotential landscape}

In order to check whether the system exhibits multiscale behavior and to validate the switch rate formula in the stochastic model, in Fig. 8 we depict the dependence of the mean first passage time $T_{i}^{n}$ on the starting point $i$ and end point $n$. As shown in the figure, the mean first passage time from the off-state stable point $i^{*}$ stays at roughly the same timescale as long as the end point $n$ lies in the on-state attractor. Similar results also hold for $T_{i}^{n^{*}}$ if $i$ lies in the offstate attractor. Therefore, it is reasonable to utilize $T_{i^{*}}^{n^{*}}$ to represent the characteristic mean switch time from the off state to the on state in the S-phase checkpoint pathway.
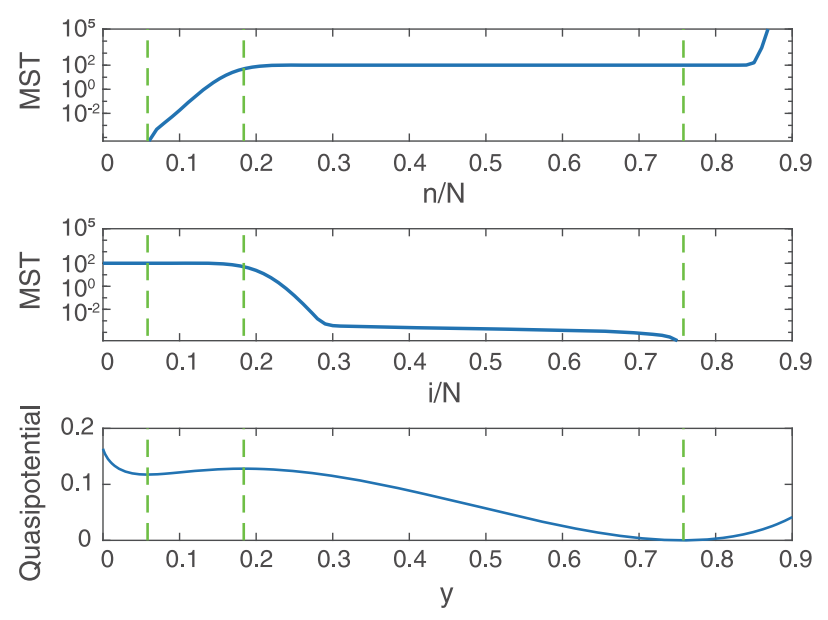

FIG. 8. Top panel: mean switch passage time $T_{i^{*}}^{n}$ with fixed low-state stable point $i^{*}$ and various end points $n$. $X$ label: relative percentage of $n$ to the total number $N$. $Y$ label: mean switch time (in logarithmic scale). Middle panel: The mean switch time $T_{i}^{n^{*}}$ for different start point $i$ with fixed $n^{*}$ as the on-state stable point. $X$ label: relative percentage of $i$ to the total number $N$. $Y$ label: mean switch time (in logarithmic scale). Bottom panel: quasipotential landscape of the system. Green lines indicate the position of the low steady state, saddle point, and high steady state. The parameters are set according to the $N=1000$ case in Sec. I of the Supplemental Material [45]. 
The results also suggest that the activation from the off state to the on state is a barrier-crossing process with different timescales of the "uphill" and "downhill" processes. Starting from the off-state stable point, the timescale of the mean first passage time increases significantly as the number of phosphorylated molecules reaches the saddle point. However, the average time will stay at the same order once the Rad53p number exceeds the saddle points.

To more vividly describe such uphill-downhill dynamics and the energy-barrier-like effect, we introduce the notion of quasipotential landscape defined as [51]

$$
\phi(x)=\lim _{V \rightarrow \infty} \frac{1}{V} \ln P_{x V}^{s s},
$$

where $P^{s s}$ is the steady-state distribution of CME. It can be calculated as [51]

$$
\phi(x)=-\int_{0}^{x} \ln \frac{R^{+}(z)}{R^{-}(z)} d z .
$$

From this expression, we know that $\phi(x)$ can also be expressed as the function of dimensionless parameters $p, q$ and total $\operatorname{Rad} 53$ concentration $R$,

$$
\phi(x)=R \tilde{\phi}\left(\frac{x}{R}\right)
$$

where

$$
\begin{aligned}
\tilde{\phi}(y)= & -\int_{0}^{y} \ln \frac{\left(q z^{2}+p\right)(1-z)}{z} d z \\
= & -y \ln \left(p+q y^{2}\right)+2 y-2 \sqrt{\frac{p}{q}} \arctan \left(\sqrt{\frac{q}{p}} y\right) \\
& +(1-y) \ln (1-y)+y \ln y .
\end{aligned}
$$

The quasipotential has a close relation with the switch rate $\lambda$ from $x_{1}$ (the off-state stable point) to $x_{2}$ (the on-state stable point) across the saddle point $x_{3}$ [60],

$$
\Delta \phi=-\lim _{V \rightarrow \infty} \frac{1}{V} \ln (\lambda),
$$

where $\Delta \phi=\phi\left(x_{3}\right)-\phi\left(x_{1}\right)$ denotes the "barrier height" of the energy landscape, which is the difference of the quasipotentials between the saddle point and the off-state stable point. Hence, we can adopt $\Delta \phi$ as a measurement to quantify the relative difficulty of the off-on switch. The larger the quasipotential barrier height is, the longer it takes to activate

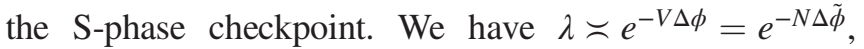
where the symbol $\asymp$ means logarithmic equivalence, i.e., $\lim _{N \rightarrow \infty} \log c_{N} / \log d_{N}=1$ if $c_{N} \asymp d_{N}$. For the sake of simplicity, in the main text, we discuss the dependency of
$\Delta \tilde{\phi}$ on $p$ and $q$ in order to explore the role of signal-induced and Rad53 autophosphorylation in the off-on switch.

\section{Validation of exponential switch time}

Next, we aim to show that the stochastic model with double-well barrier-crossing dynamics can successfully explain the exponentially distributed switch time (i.e., the linear region in the survival curve in Fig. 3 of the main text).

Recall the definition of the first passage time for Rad53p number $i$ to $n$,

$$
\tau_{i}^{n}=\inf \left\{t \mid t>0, X_{t}=n, X_{0}=i\right\},
$$

where $X_{t}$ denotes the number of Rad53p at time $t$. We can derive the equation for the distribution of $\tau_{i}^{n}$ following similar derivations for its expectation. If we define the survival probability $S_{i}^{n}(t)=\mathbb{P}\left(\tau_{i}^{n}>t\right)$, then we have

$$
\begin{aligned}
S_{i}^{n}(t)= & w_{i} \Delta t S_{i-1}^{n}(t-\Delta t)+u_{i} \Delta t S_{i+1}^{n}(t-\Delta t) \\
& +\left(1-\left(u_{i}+w_{i}\right) \Delta t\right) S_{i}^{n}(t-\Delta t)+o(\Delta t)
\end{aligned}
$$

and consequently, as $\Delta t \rightarrow 0$,

$$
\begin{aligned}
\frac{d S_{i}^{n}(t)}{d t} & =w_{i} S_{i-1}^{n}(t)-\left(u_{i}+w_{i}\right) S_{i}^{n}(t)+u_{i} S_{i+1}^{n}(t), \\
i & =1,2, \ldots, \quad n-1,
\end{aligned}
$$

with the initial and boundary conditions

$$
\begin{aligned}
& S_{i}(0)=1, \quad i=0,2, \ldots, n-1, \\
& S_{n}(t)=0 .
\end{aligned}
$$

Based on the parameters determined in the previous section, the $\ln S_{i^{*}}^{n^{*}}(t)$ curve is plotted in Figs. 9 and 4(b) of the main text [i.e., the $\ln R_{\text {off }}(t)$ curve], where $i^{*}$ and $n^{*}$ correspond to the number of Rad53p of the off-state stable point and the on-state stable point, respectively. We observe that it is a linear function for different concentrations of $\mathrm{HU}$, indicating that $\tau_{i^{*}}^{n^{*}}$ approximately follows the exponential distribution.

From the obtained results of $S_{i}^{n}$, we are also able to obtain the distribution of maximum Rad53p molecules $N_{m}$ within a certain time $t\left[N_{m}(t)=\max _{s \leq t} X_{s}\right]$, which is the theoretical counterpart of MFGR in the single-cell experiment. Since $\mathbb{P}\left(N_{m}<n \mid X_{0}=i^{*}\right)=\mathbb{P}\left(\tau_{i^{*}}^{n}>t\right)=S_{i^{*}}^{n}$, we have that $\mathbb{P}\left(N_{m}=n \mid X_{0}=i^{*}\right)=S_{i^{*}}^{n+1}-S_{i^{*}}^{n}$.

\section{Discussion on mutant strains}

By adjusting the parameters in the quantitative model, we are able to predict the phenomenon in other mutant strains. Below, we first discuss the case with $\operatorname{Rad} 53$ autophosphorylation mutant strains, whose parameter $q$ 

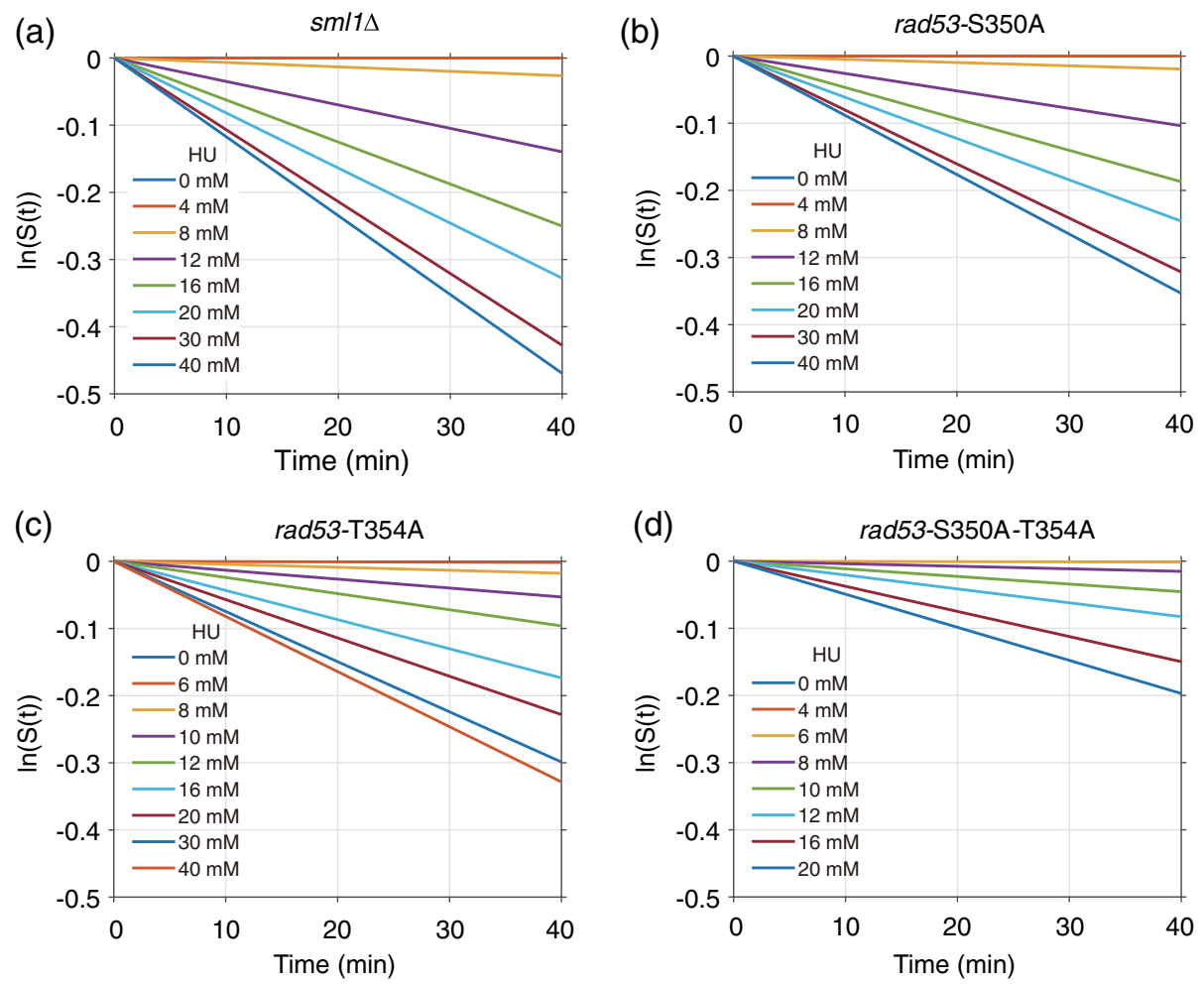

FIG. 9. The theoretical switch time $\tau_{i^{*}}^{n^{*}}$ follows the exponential distribution for $\operatorname{sml1\Delta }$ (a), S350A (b), rad53-T354A (c), and rad53-S350A-T354A (d) strains. $X$ label: time (min). $Y$ label: logarithm of survival probability.

differs from $s m l \Delta$ cells. Then, we turn to the analysis of the S-phase checkpoint pathway activation behavior in the Rad53 overexpression cells. Finally, we discuss the effect of extrinsic noise in the S-phase checkpoint activation model.

\section{a. Rad53 autophosphorylation mutant strains}

When certain autophosphorylation sites of $\operatorname{Rad} 53$ are mutated, the corresponding parameter $k_{2}$ will be reduced.
As discussed in the main text and Supplemental Material [45], although the off-on switch still exists in the rad53-S350A mutant, the switch rate in checkpoint activation has decreased. As shown in Fig. 10, if we continue decreasing the value of $q$, then a threshold effect of $\mathrm{HU}$ doses in stimulating the off-on switch will arise: Under the low HU concentration treatment, the bimodal distribution of MFGR may become invisible due to the negligible (a)

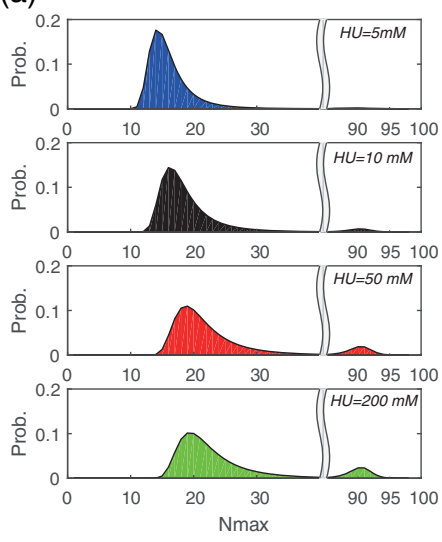

(b)

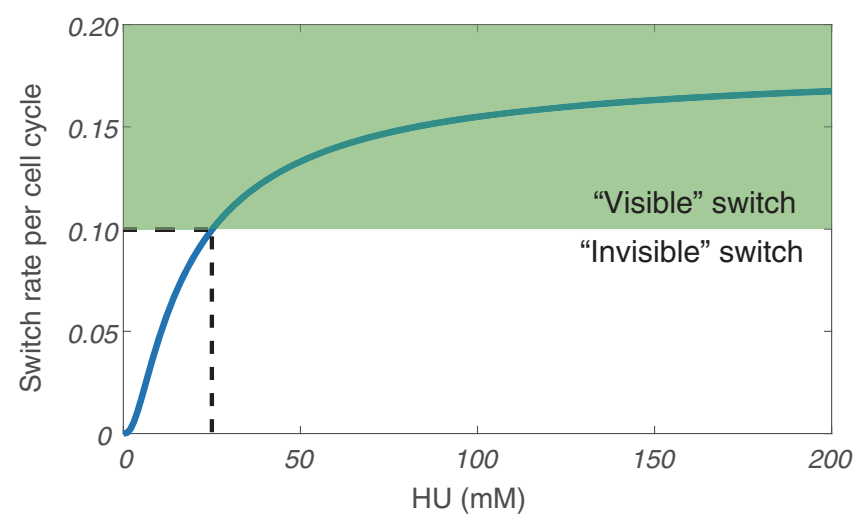

FIG. 10. Threshold effect of HU doses in the off-on switch of autophosphorylation site mutant strains. (a) Probability distribution of $N_{m}(t=70 \mathrm{~min}$ ) (maximum within $70 \mathrm{~min}$ ) under different HU doses. The bimodal distribution can hardly be observed for HU $=5$ $\mathrm{mM}$ and 10-mM doses. (b) Off-on switch rate under different HU doses. $X$ label: concentration of HU stimulation. $Y$ label: switch rate per cell cycle (taking $100 \mathrm{~min}$, for simplicity). The threshold of the switch rate is chosen such that $10 \%$ of the cells can be activated during the cell cycle. 

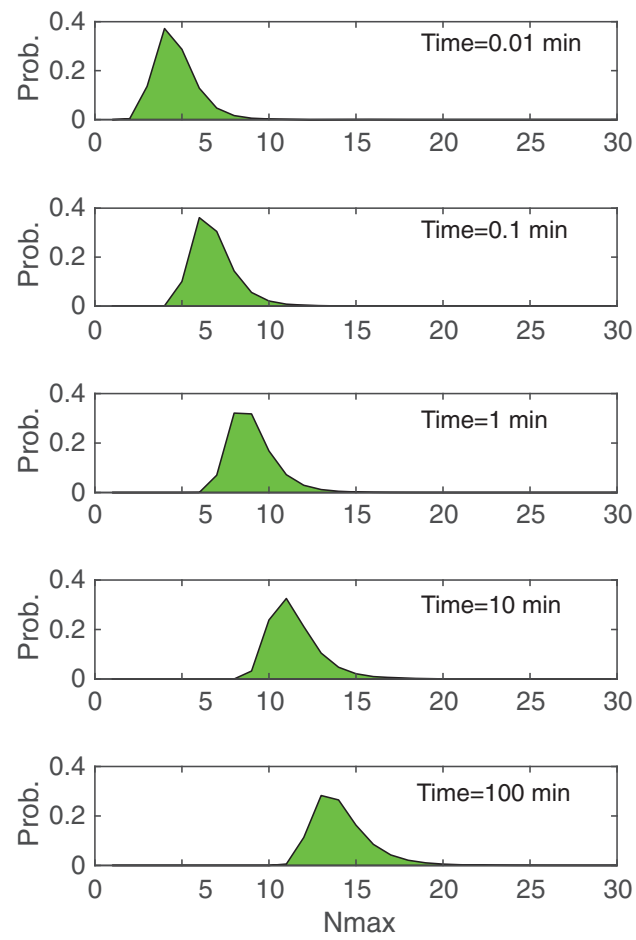

FIG. 11. Shift of the $N_{m}$ probability distribution in unistable autophosphorylation mutant strains with the progress of time.

off-on switch rate in the experimental timescale. For instance, if the HU stimulation is below $25 \mathrm{mM}$, then the switch rate is below 0.1 per cell cycle (approximately $100 \mathrm{~min}$ ), and over $90 \%$ of the cells remain inactivated within the whole cell-cycle time, whose on-state can hardly be probed in the $N_{m}$ distribution. Only when the HU doses exceed the threshold concentration can we observe the bimodal distribution of $N_{m}$ (or the experimental MFGR).

If we further decrease the value of $q$ such that the parameter setting is beyond the bistable region, then only the unimodal distribution of $N_{m}$ can be obtained. With the progress of time, we observe the rightward shift of the $N_{m}$ probability distribution (Fig. 11), reminiscent of the graded response described in the main text.

\section{b. Rad53 overexpression strains}

If the Rad53 protein is overexpressed in the budding yeast cell, we can assume that the total Rad53 concentration becomes $s R(s>1)$. Then, the dimensionless parameters $q$ and $N$ should be multiplied by factors $s^{2}$ and $s$, respectively.

To explore the effect of Rad53 overexpression, in Fig. 12 we depict the switch rate as a function of overexpression scale $s$. The original parameters for $s=1$ are chosen as the settings for $\operatorname{sml} l \Delta$ strains, and the parameter $p$ is fixed at the value corresponding to $\mathrm{HU}=0.7 \mathrm{mM}$. We find that when the overexpression scale is beyond a certain threshold, the switch rate will escalate dramatically with the
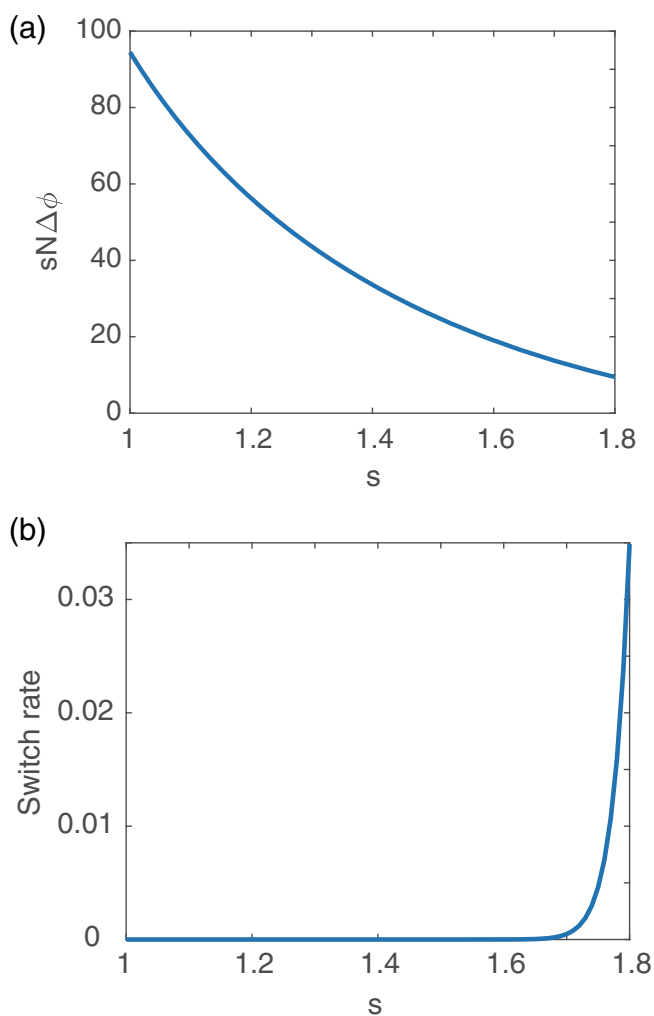

FIG. 12. Simulation results for Rad53 overexpression strains. (a) Dependence of the switch rate on the overexpression scale $s$. (b) Relationship between $N \Delta \tilde{\phi}$ and $s$. The parameters for $s=1$ are chosen to be the same as the setting for $\operatorname{sml} l \Delta$ strains, and the concentration of $\mathrm{HU}$ stimulation is set as $0.7 \mathrm{mM}$. The saturation switch rate observed in the experiment is around $0.01 \mathrm{~min}^{-1}$.

increase of $s$, eventually exceeding the maximum switch rate obtained from the experimental survival curve. This result indicates that the $\mathrm{S}$-phase checkpoint will activate in these overexpression strains even when the replication stress signal is very weak.

The differences between overexpression strains and the results on the sensitivity analysis about $N$ in Sec. I of the Supplemental Material [45] can be understood from both deterministic and stochastic perspectives.

In deterministic models, when we discuss the sensitivity of parameter $N$, the other parameters (dimensional $k_{1}$, $k_{2}, k_{m}, R$ and dimensionless $p, q$ ) are all held as constants. Hence, the steady state of ODEs does not change with different choices of $N$. However, in the overexpression strains, the total $\operatorname{Rad} 53$ concentration $R$ (therefore, $q$ ) is rescaled, resulting in the change of stable and saddle points of ODE systems with respect to the overexpression factor $s$.

In stochastic models, the key difference between the two regimes is whether the volume $V$ is held constant. For the sensitivity analysis, $V$ is adjusted with $N$ to keep $R$ constant; however, in the overexpression strains, $V$ is fixed and $N$ is changed according to the overexpression of $R$. Therefore, $u_{n}(V)$ 's in the two regimes are different. When calculating the switch rate, the choice of end point $n^{*}$ is also 
different because of the distinctions in the ODE systems. These differences resolve the seemingly paradoxical results that, in the sensitivity analysis, the switch rate decreases with the increase of $N$, while the models of overexpression strains suggest that, with more Rad53 molecules, the off-on switch tends to be boosted. In terms of the quasipotential point of view, for sensitivity analysis, the key variable is the system size $V$, and we have $\lambda(V) \asymp e^{-V \Delta \phi}=e^{-N(V) \Delta \tilde{\phi}}$, where $N(V)=R V$ and $\Delta \tilde{\phi}$ is independent of $V$. Hence, the increase in $N$, which is the result of increasing $V$, will be accompanied by a decrease of the switch rate. On the other hand, in the overexpression models, the key variable is the overexpression scale $s$, and we have $\lambda(s) \asymp e^{-N(s) \Delta \tilde{\phi}(p, q(s))}$. Here, $N(s)$ is a linear function of $s$, while $\Delta \tilde{\phi}(p, q(s))$ is a decreasing function of $s$. Its product, as plotted in Fig. 12(b), is also a decreasing function of $s$, explaining the relationship between the overexpression scale and the switch rate calculated in Fig. 12(a).

\section{Effect of molecule number fluctuations on switch rates}

Our previous mathematical model does not take into account the effect of extrinsic noise on the number of total $\operatorname{Rad} 53$ molecules. When the extrinsic noise is considered, the corresponding $q$ value of the fitting parameter will increase slightly. In order to simulate the influence of extrinsic noise on cells, we suppose the $N$ value (total number of phosphorylated and unphosphorylated Rad53 molecules) obeys Gaussian distribution. Considering that $N$ is around $10^{3}$ [65], we take the standard deviation of the $N$ value to be 100 and make 1000 calculations; then, the fitting curve of the extrinsic noise correction could be obtained as well as $q$.

The $q$ value is the only variable among different mutant strains that determines the strength of autophosphorylation of Rad53 and also determines the switch rate from the off state to the on state. After considering extrinsic noise, the $q$ values of $s m l 1 \Delta$ strains and various mutant strains are 5.410 $(\operatorname{sml1\Delta }), 5.400$ (rad53-S350A), 5.385 ( $r a d 53-T 354 \mathrm{~A})$, and 5.375 ( $r a d 53-S 350 A-T 354 A)$, respectively (Fig. 13), which is slightly higher than the switch rate fitted without extrinsic noise (5.380 of $s m l 1 \Delta, 5.360$ of rad53-S350A, 5.355 of rad53-T354A, and 5.345 of $\mathrm{rad53}-\mathrm{S350 \textrm {A } -}$ T354A). In addition, the rad53-S350A strain was selected to demonstrate the relationship between the standard deviation of $N$ obeying Gaussian distribution and the fitted switch rate in Fig. S3.

\section{Effect of heterogeneous growth on switch rates}

Another source of noise that affects the transitions may originate from the fact that the yeast cells grow with the heterogeneous speed during the cell cycle, while the growth dilutes Rad53 and therefore induces the effect of growthmediated feedback [62].

According to the microscopic interpretation of chemical reactions [73], the parameter $q=\left(k_{2} N^{2} / k_{m} V^{2}\right)$ in the stochastic model is subject to the influence of the system size $V$ and the associated growth heterogeneity effect. Similar to the analysis on molecular number fluctuation, here we assume that $q$ follows the Gaussian distribution. As shown in Fig. 14, after taking the effect of heterogeneous growth into account, the stochastic model can still fit the (a)

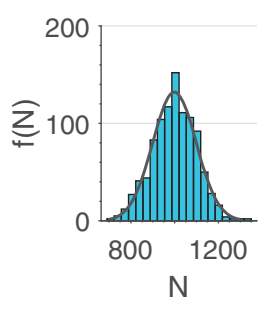

(c)

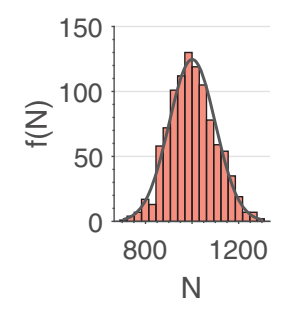

$s m / 1 \Delta$

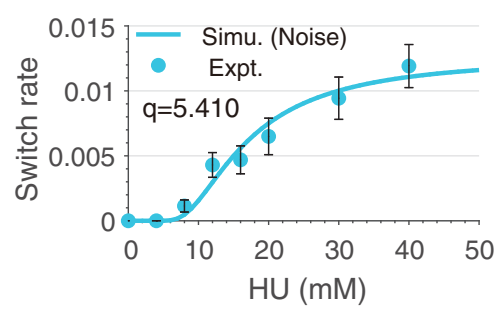

rad53-T354A

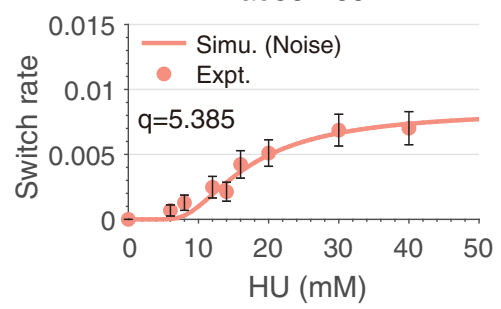

(b)

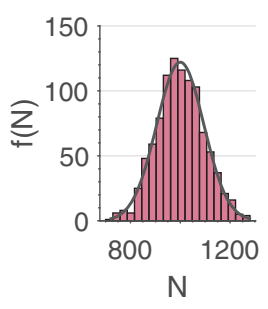

(d)

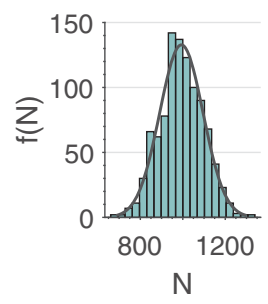

rad53-S350A

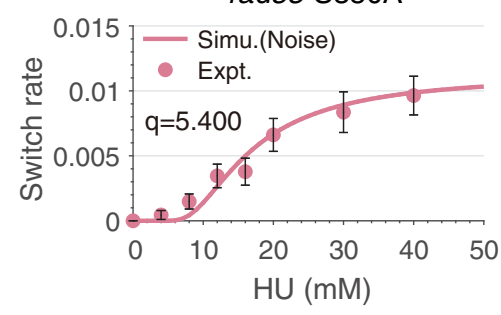

rad53-S350A-T354A

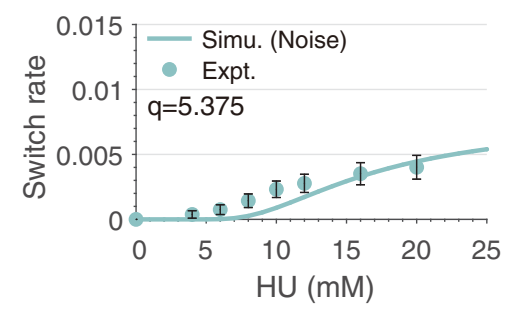

FIG. 13. Switch rate of $\operatorname{sml1\Delta }$ (a), rad53-S350A (b), rad53-T354A (c), and rad53-S350A-T354A (d) strains after considering the extrinsic noise. The left panel of each subfigure denotes the distribution of $N$ values (number of all Rad53 molecules) in our simulations, which obeys a Gaussian distribution with a standard deviation of 100. The right panel of each subfigure denotes the corresponding switch rate. The switch rate of the WT strain is similar to the $\operatorname{sml1\Delta }$ strain, as shown in the Supplemental Material [45]. 
(a)

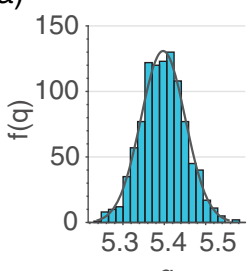

q

(c)

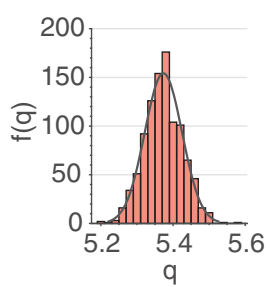

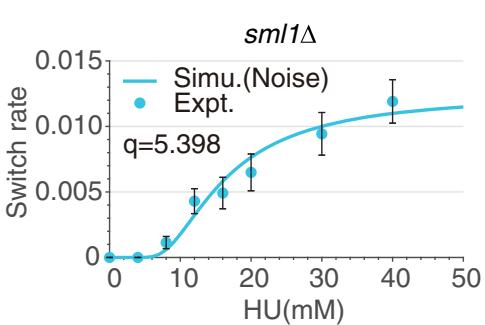

(b)

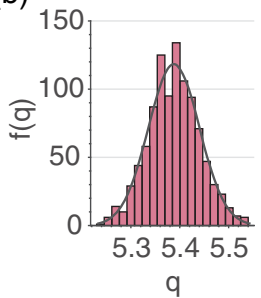

(d)
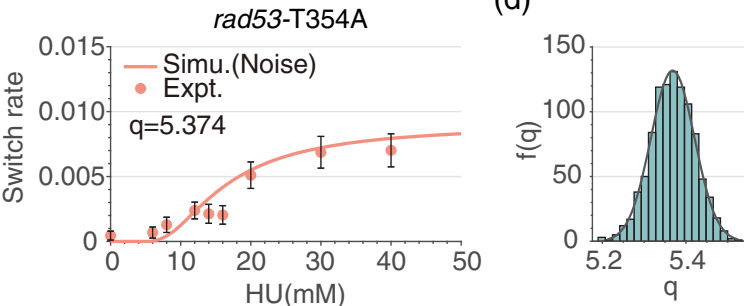
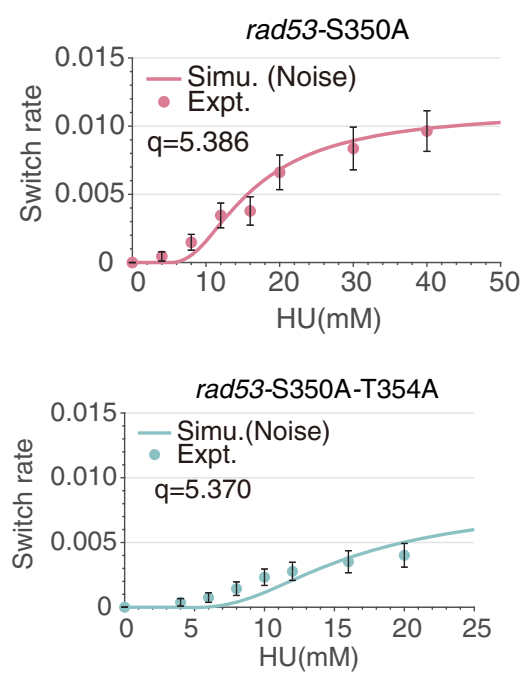

FIG. 14. Switch rate of $\operatorname{sml1\Delta }$ (a), rad53-S350A (b), rad53-T354A (c), and rad53-S350A-T354A (d) strains after considering the heterogeneous growth effect. The left panel of each subfigure denotes the distribution of parameter $q$ (parameter $q$ is related to the growth heterogeneity effect) in our simulations, which obeys a Gaussian distribution. The right panel of each subfigure denotes the corresponding switch rate. The switch rate of the WT strain is similar to the $s m l 1 \Delta$ strain, as shown in the Supplemental Material [45].

experimental switch rate, but with larger $q$ values for each strain.

\section{Analysis on an alternative hypothesis of nonlinear RNR response}

In the main text, we focused on the nonlinear phosphorylation of Rad53 triggered by the DNA damage signal as the key mechanism for the noise-induced transition, and we also used experimental evidence from mutant strains to validate this theory. Here, we inspect an alternative explanation about nonlinear RNR complex response and derive its inconsistency with experimental findings.

In such an alternative theory, we assume that the RNR complex molecules have either active or inactive forms, and the activation function takes the nonlinear form. Only the active RNR can function in the synthesis of DNA and regulate the pool level of dNTP. To model the inhibition of the RNR complex by HU, we have

$$
\begin{aligned}
\frac{d\left[\mathrm{RNR}_{\mathrm{in}}\right]}{d t}= & h_{1}\left(\left[\mathrm{RNR}_{\mathrm{in}}\right] ;[\mathrm{HU}]\right)+k_{+}\left(R_{T}-\left[\mathrm{RNR}_{\mathrm{in}}\right]\right) \\
& -k_{-}\left[\mathrm{RNR}_{\mathrm{in}}\right],
\end{aligned}
$$

where $R_{T}$ denotes the total concentration of the RNR complex. Here, $h_{1}\left(\left[\mathrm{RNR}_{\text {in }}\right] ;[\mathrm{HU}]\right)$ models the nonlinear effect of RNR inhibition, where a simple form analogous to our previous $\operatorname{Rad} 53$ model would be $h_{1}(x ;[\mathrm{HU}])=$ $g([\mathrm{HU}]) x^{2}\left(R_{T}-x\right), g([\mathrm{HU}])=p_{0}[\mathrm{HU}]^{n} /\left(K_{\mathrm{HU}}^{n}+[\mathrm{HU}]^{n}\right)$. The steady-state solution can be bistable, forming two metastable states denoted as LOW (major inactive RNR and minor active RNR) and HIGH (major active RNR and minor inactive RNR), which lead to low or high levels of dNTP in the single cell, respectively.
Also, the phosphorylation of $\operatorname{Rad} 53$ is assumed to be linear, responding to the signal of dNTP depletion and accumulation of single-strand DNA:

$\frac{d\left[\operatorname{Rad} 53_{p}\right]}{d t}=h_{2}\left(\left[\mathrm{RNR}_{\mathrm{in}}\right]\right)\left(R-\left[\operatorname{Rad} 53_{p}\right]\right)-k_{3}\left[\operatorname{Rad} 53_{p}\right]$,

where $h_{2}(\cdot)$ is an increasing function of the inactive RNR level.

Lastly, the total number of the RNR complex is transcriptionally regulated by phosphorylated Rad53,

$$
\frac{d R_{T}}{d t}=h_{3}\left(\left[\operatorname{Rad} 53_{p}\right]\right)-k_{4} R_{T} .
$$

Here, $h_{3}(\cdot)$ is the increasing function, and it can be commonly assumed with the Hill-function form.

To explain the transition during the activation process of the S-phase checkpoint, we assume the phosphorylation reaction in Eq. (C2) is fast, while the transcription and translation processes in Eq. (C3) are slow. Hence, we can assume the dynamical equilibrium $\left[\operatorname{Rad} 53_{p}\right]=$ $R h_{2}\left(\left[\mathrm{RNR}_{\text {in }}\right]\right) /\left(k_{3}+h_{2}\left(\left[\mathrm{RNR}_{\text {in }}\right]\right)\right)$, and $R_{T}$ remains constant in a short time period. Therefore, during the activation process, the number of phosphorylated Rad53 is actually the indicator of the inactive RNR complex level, and the dynamics are fully restricted by the state-switch process of the RNR complex $(\mathrm{C} 1)$. Under such a hypothesis, the observed bimodal distribution and relevant transition process might result from the nonlinear inhibition of RNR by $\mathrm{HU}$, instead of the autophosphorylation of Rad53. 
While the hypothesis seems plausible to explain the bimodal distribution, we find that it is insufficient to account for other key experimental facts. Our experiments suggest that KRK-EDE mutations in autophosphorylation sites of $\operatorname{Rad} 53$, which only affect the intensity in $h_{2}(\cdot)$, could indeed result in the unimodal distribution of MFGR. However, without the assumptions about Rad53 autophosphorylation, the distribution cannot be solely explained by the nonlinear dynamics of the RNR complex. We also observed a significant reduction of the S-phase checkpoint switch rate in mutant strains of Rad53 autophosphorylation sites (Fig. 4), which cannot be predicted by the RNR nonlinear inhibition model. Combined with further experimental evidence $[74,75]$ on the Rad53 autophosphorylation process, we conclude that the nonlinearity in the Rad53 response plays an important role in the stochastic transition mechanism of S-phase checkpoint activation.

\section{APPENDIX D: STATISTICAL ANALYSIS OF RATE ESTIMATION}

As discussed in the main text, the linear region of the survival curves (whose $Y$ label is in the logarithmic scale) indicates that the off-on switch time follows the exponential distribution with rate $\lambda$ in probability theory. To estimate the relevant switch rate, we conduct regression for the linear part of the survival curve, and we regard the minus value of the slope as the experimental switch rate $\hat{\lambda}$. In the Supplemental Material [45], we discuss the rationale of this estimation method and build up the confidence interval of such an estimation, which is guaranteed by the following theorems (see Sec. II of the Supplemental Material [45] for details).

Theorem 1. The estimator $\hat{\lambda}$ is consistent, i.e., $\hat{\lambda} \stackrel{a . s .}{\rightarrow} \lambda$ as sample size $N_{\text {sample }} \rightarrow \infty$.

Theorem 2. When the linear regression is feasible, the mean deviation between the estimator and true parameter $\lambda$ converges at order $O\left(N_{\text {sample }}^{-\frac{1}{2}}\right)$.

[1] U. Alon, An Introduction to Systems Biology: Design Principles of Biological Circuits (Chapman and Hall/ CRC, Boca Raton, 2007).

[2] J.E. Ferrell and S.H. Ha, Ultrasensitivity Part III: Cascades, Bistable Switches, and Oscillators, Trends Biochem. Sci. 39, 612 (2014).

[3] G. Balázsi, A. van Oudenaarden, and J. J. Collins, Cellular Decision Making and Biological Noise: From Microbes to Mammals, Cell 144, 910 (2011).

[4] M. B. Elowitz, A. J. Levine, E. D. Siggia, and P. S. Swain, Stochastic Gene Expression in a Single Cell, Science 297, 1183 (2002).

[5] W. J. Blake, M. Kaern, C. R. Cantor, and J. J. Collins, Noise in Eukaryotic Gene Expression, Nature (London) 422, 633 (2003).
[6] R. A. Kellogg and S. Tay, Noise Facilitates Transcriptional Control under Dynamic Inputs, Cell 160, 381 (2015).

[7] K. Hayashi, S. M. C. de Sousa Lopes, F. Tang, and M. A. Surani, Dynamic Equilibrium and Heterogeneity of Mouse Pluripotent Stem Cells with Distinct Functional and Epigenetic States, Cell Stem Cell 3, 391 (2008).

[8] T. M. Norman, N. D. Lord, J. Paulsson, and R. Losick, Stochastic Switching of Cell Fate in Microbes, Annu. Rev. Microbiol. 69, 381 (2015).

[9] J. E. Purvis, K. W. Karhohs, C. Mock, E. Batchelor, A. Loewer, and G. Lahav, p53 Dynamics Control Cell Fate, Science 336, 1440 (2012).

[10] S. L. Spencer, S. Gaudet, J. G. Albeck, J. M. Burke, and P. K. Sorger, Non-genetic Origins of Cell-to-Cell Variability in Trail-Induced Apoptosis, Nature (London) 459, 428 (2009).

[11] J. Roux, M. Hafner, S. Bandara, J. J. Sims, H. Hudson, D. N. Chai, and P. K. Sorger, Fractional Killing Arises from Cellto-Cell Variability in Overcoming a Caspase Activity Threshold, Mol. Syst. Biol. 11, 803 (2015).

[12] M. Heltberg, R. A. Kellogg, S. Krishna, S. Tay, and M. H. Jensen, Noise Induces Hopping between NF-kB Entrainment Modes, Cell Syst. 3, 532 (2016).

[13] J. Yao, A. Pilko, and R. Wollman, Distinct Cellular States Determine Calcium Signaling Response, Mol. Syst. Biol. 12, 894 (2016).

[14] J. M. Raser and E. K. O'Shea, Control of Stochasticity in Eukaryotic Gene Expression, Science 304, 1811 (2004).

[15] K. N. Rybakova, F. J. Bruggeman, A. Tomaszewska, M. J. Moné, C. Carlberg, and H. V. Westerhoff, Multiplex Eukaryotic Transcription (In)activation: Timing, Bursting and Cycling of a Ratchet Clock Mechanism, PLoS Comput Biol. 11, e1004236 (2015).

[16] P. J. Choi, L. Cai, K. Frieda, and X. S. Xie, A Stochastic Single-Molecule Event Triggers Phenotype Switching of a Bacterial Cell, Science 322, 442 (2008).

[17] L. Zhang, K. Radtke, L. Zheng, A. Q. Cai, T. F. Schilling, and Q. Nie, Noise Drives Sharpening of Gene Expression Boundaries in the Zebrafish Hindbrain, Mol. Syst. Biol. 8, 613 (2012).

[18] D. Nevozhay, R. M. Adams, E. Van Itallie, M. R. Bennett, and G. Balázsi, Mapping the Environmental Fitness Landscape of a Synthetic Gene Circuit, PLoS Comput Biol. 8, e1002480 (2012).

[19] X. Fang, Q. Liu, C. Bohrer, Z. Hensel, W. Han, J. Wang, and J. Xiao, Cell Fate Potentials and Switching Kinetics Uncovered in a Classic Bistable Genetic Switch, Nat. Commun. 9, 2787 (2018).

[20] D. O. Morgan, The Cell Cycle: Principles of Control (New Science Press, London, 2007).

[21] J. Bartek, C. Lukas, and J. Lukas, Checking on DNA Damage in S Phase, Nat. Rev. Mol. Cell Biol. 5, 792 (2004).

[22] N. Nordlund and P. Reichard, Ribonucleotide Reductases, Annu. Rev. Biochem. 75, 681 (2006).

[23] N. C. Hoch, E. S. W. Chen, R. Buckland, S. C. Wang, A. Fazio, A. Hammet, A. Pellicioli, A. Chabes, M. D. Tsai, and J. Heierhorst, Molecular Basis of the Essential S Phase Function of the Rad53 Checkpoint Kinase, Mol. Cell. Biol. 33, 3202 (2013).

[24] J. Melo and D. Toczyski, A Unified View of the DNADamage Checkpoint, Curr. Opin. Cell Biol. 14, 237 (2002). 
[25] B. B. Zhou and S. J. Elledge, The DNA Damage Response: Putting Checkpoints in Perspective, Nature (London) 408, 433 (2000).

[26] J. Bartek and J. Lukas, Chk1 and Chk2 Kinases in Checkpoint Control and Cancer, Cancer Cell 3, 421 (2003).

[27] M. P. Longhese, M. Clerici, and G. Lucchini, The S-Phase Checkpoint and Its Regulation in Saccharomyces cerevisiae, Mutat. Res. 532, 41 (2003).

[28] B. Pardo, L. Crabbe, and P. Pasero, Signaling Pathways of Replication Stress in Yeast, FEMS Yeast Res. 17, fow 101 (2017).

[29] A. Koc, L. J. Wheeler, C. K. Mathews, and G. F. Merrill, Hydroxyurea Arrests DNA Replication by a Mechanism that Preserves Basal dNTP Pools, J. Biol. Chem. 279, 223 (2004).

[30] G. M. Alvino, D. Collingwood, J. M. Murphy, J. Delrow, B. J. Brewer, and M. K. Raghuraman, Replication in Hydroxyurea: It's a Matter of Time, Mol. Cell. Biol. 27, 6396 (2007).

[31] A. R. Paterson and A. I. Simpson, Inhibition of Ribonucleoside Metabolism in Ehrlich Ascites Tumor Cells by Purine Analogue Ribonucleosides, Can. J. Biochem. 43, 1701 (1965).

[32] A. Singh and Y. J. Xu, The Cell Killing Mechanisms of Hydroxyurea, Genes 7, 99 (2016).

[33] T. J. Berens and D. P. Toczyski, Colocalization of Mec1 and Mrc1 Is Sufficient for Rad53 Phosphorylation in Vivo, Mol. Biol. Cell 23, 1058 (2012).

[34] S. H. Chen and H. L. Zhou, Reconstitution of Rad53 Activation by Mecl through Adaptor Protein Mrc1, J. Biol. Chem. 284, 18593 (2009).

[35] M. Clerici, C. Trovesi, A. Galbiati, G. Lucchini, and M. P. Longhese, Mecl/ATR Regulates the Generation of SingleStranded DNA that Attenuates Tell/ATM Signaling at DNA Ends, EMBO J. 33, 198 (2014).

[36] M. F. Schwartz, J. K. Duong, Z. Sun, J. S. Morrow, D. Pradhan, and D.F. Stern, Rad9 Phosphorylation Sites Couple Rad53 to the Saccharomyces cerevisiae DNA Damage Checkpoint, Mol. Cell 9, 1055 (2002).

[37] F. D. Sweeney, F. Yang, A. Chi, J. Shabanowitz, D. F. Hunt, and D. Durocher, Saccharomyces cerevisiae Rad9 Acts as a Mecl Adaptor to Allow Rad53 Activation, Curr. Biol. 15, 1364 (2005).

[38] S. H. Chen, M. B. Smolka, and H. Zhou, Mechanism of Dun1 Activation by Rad53 Phosphorylation in Saccharomyces cerevisiae, J. Biol. Chem. 282, 986 (2007).

[39] B. L. Andreson, A. Gupta, B. P. Georgieva, and R. Rothstein, The Ribonucleotide Reductase Inhibitor, Smll, Is Sequentially Phosphorylated, Ubiquitylated and Degraded in Response to DNA Damage, Nucleic Acids Res. 38, 6490 (2010).

[40] A. Chabes, V. Domkin, and L. Thelander, Yeast Sml1, a Protein Inhibitor of Ribonucleotide Reductase, J. Biol. Chem. 274, 36679 (1999).

[41] M. X. Huang, Z. Zhou, and S. J. Elledge, The DNA Replication and Damage Checkpoint Pathways Induce Transcription by Inhibition of the Crt1 Repressor, Cell 94, 595 (1998).

[42] Y. D. Lee, J. Wang, J. Stubbe, and S. J. Elledge, Difl Is a DNA-Damage-Regulated Facilitator of Nuclear Import for Ribonucleotide Reductase, Mol. Cell 32, 70 (2008).
[43] G. Guillemain, E. Ma, S. Mauger, S. Miron, R. Thai, R. Guerois, F. Ochsenbein, and M. C. Marsolier-Kergoat, Mechanisms of Checkpoint Kinase Rad53 Inactivation after a Double-Strand Break in Saccharomyces cerevisiae, Mol. Cell. Biol. 27, 3378 (2007).

[44] C. Leroy, S. E. Lee, M. B. Vaze, F. Ochsenbein, R. Guerois, J. E. Haber, and M. C. Marsolier-Kergoat, PP2C phosphatases Ptc2 and Ptc3 Are Required for DNA Checkpoint Inactivation after a Double-Strand Break, Mol. Cell 11, 827 (2003).

[45] See Supplemental Material at http://link.aps.org/ supplemental/10.1103/PhysRevX.11.011004 for the additional data and analysis.

[46] B. A. Evert, T. B. Salmon, B. Song, J. Liu, W. Siede, and P. W. Doetsch, Spontaneous DNA Damage in Saccharomyces cerevisiae Elicits Phenotypic Properties Similar to Cancer Cells, J. Biol. Chem. 279, 22585 (2004).

[47] J. F. Lawless, Statistical Models and Methods for Lifetime Data, 2nd ed. (John Wiley \& Sons, Hoboken, 2003).

[48] S. Fiorani, G. Mimun, L. Caleca, D. Piccini, and A. Pellicioli, Characterization of the Activation Domain of the Rad53 Checkpoint Kinase, Cell Cycle 7, 493 (2008).

[49] J. L. Ma, S. J. Lee, J. K. Duong, and D. F. Stern, Activation of the Checkpoint Kinase Rad53 by the Phosphatidyl Inositol Kinase-like Kinase Mec1, J. Biol. Chem. 281, 3954 (2006).

[50] E. S. W. Chen, N. C. Hoch, S. C. Wang, A. Pellicioli, J. Heierhorst, and M.D. Tsai, Use of Quantitative Mass Spectrometric Analysis to Elucidate the Mechanisms of Phospho-priming and Auto-activation of the Checkpoint Kinase Rad53 in Vivo, Mol. Cell Proteomics 13, 551 (2014).

[51] H. Ge and H. Qian, Thermodynamic Limit of a Nonequilibrium Steady State: Maxwell-Type Construction for a Bistable Biochemical System, Phys. Rev. Lett. 103, 148103 (2009).

[52] C. Jin, X. Yan, and F. Li, Non-equilibrium and Stochasticity Influence the Activation Process of the Yeast DNA Damage Pathway, Sci. China Phys. Mech. 61, 028721 (2018).

[53] S. Ghaemmaghami, W. Huh, K. Bower, R. W. Howson, A. Belle, N. Dephoure, E. K. O'Shea, and J. S. Weissman, Global Analysis of Protein Expression in Yeast, Nature (London) 425, 737 (2003).

[54] S. Gandy, A. J. Czernik, and P. Greengard, Phosphorylation of Alzheimer Disease Amyloid Precursor Peptide by Protein Kinase $C$ and Ca2+/Calmodulin-Dependent Protein Kinase II, Proc. Natl. Acad. Sci. U.S.A. 85, 6218 (1988).

[55] C. Lv, X. Li, F. Li, and T. Li, Constructing the Energy Landscape for Genetic Switching System Driven by Intrinsic Noise, PLoS One 9, e88167 (2014).

[56] P. Zhou and T. Li, Construction of the Landscape for Multistable Systems: Potential Landscape, Quasi-potential, A-type Integral and Beyond, J. Chem. Phys. 144, 094109 (2016).

[57] S. Huang, F. Li, J. Zhou, and H. Qian, Processes on the Emergent Landscapes of Biochemical Reaction Networks and Heterogeneous Cell Population Dynamics: Differentiation in Living Matters, J. R. Soc. Interface 14 (2017).

[58] J. Wang, K. Zhang, L. Xu, and E. Wang, Quantifying the Waddington Landscape and Biological Paths for 
Development and Differentiation, Proc. Natl. Acad. Sci. U.S.A. 108, 8257 (2011).

[59] H. Qian, P. Ao, Y. H. Tu, and J. Wang, A Framework Towards Understanding Mesoscopic Phenomena: Emergent Unpredictability, Symmetry Breaking and Dynamics across Scales, Chem. Phys. Lett. 665, 153 (2016).

[60] Y. Zhang, H. Ge, and H. Qian, One-Dimensional BirthDeath Process and Delbrück-Gillespie Theory of Mesoscopic Nonlinear Chemical Reactions, Stud. Appl. Math. 129, 328 (2012).

[61] M. Acar, A. Becskei, and A. van Oudenaarden, Enhancement of Cellular Memory by Reducing Stochastic Transitions, Nature (London) 435, 228 (2005).

[62] D. A. Charlebois, K. Hauser, S. Marshall, and G. Balázsi, Multiscale Effects of Heating and Cooling on Genes and Gene Networks, Proc. Natl. Acad. Sci. U.S.A. 115, E10797 (2018).

[63] M. V. Day, On the Exponential Exit Law in the Small Parameter Exit Problem, Stochastics 8, 297 (1983).

[64] P. Hänggi, P. Talkner, and M. Borkovec, Reaction-Rate Theory: Fifty Years after Kramers, Rev. Mod. Phys. 62, 251 (1990).

[65] www.bionumbers.org.

[66] G. M. Suel, J. Garcia-Ojalvo, L. M. Liberman, and M. B. Elowitz, An Excitable Gene Regulatory Circuit Induces Transient Cellular Differentiation, Nature (London) 440, 545 (2006).

[67] H. Maamar, A. Raj, and D. Dubnau, Noise in Gene Expression Determines Cell Fate in Bacillus subtilis, Science 317, 526 (2007).
[68] X. Liu, X. Wang, X. Yang, S. Liu, L. Jiang, Y. Qu, L. Hu, Q. Ouyang, and C. Tang, Reliable Cell Cycle Commitment in Budding Yeast Is Ensured by Signal Integration, eLife 4, e03977 (2015).

[69] C. Luo, L. Jiang, S. Liang, Q. Ouyang, H. Ji, and Y. Chen, High-Throughput Microfluidic System for Monitoring Diffusion-Based Monolayer Yeast Cell Culture over Long Time Periods, Biomed. Microdevices 11, 981 (2009).

[70] X. Yang, K. Y. Lau, V. Sevim, and C. Tang, Design Principles of the Yeast G1/S Switch, PLoS Biol. 11, e1001673 (2013).

[71] Y. Xu, M. Davenport, and T. J. Kelly, Two-Stage Mechanism for Activation of the DNA Replication Checkpoint Kinase Cds1 in Fission Yeast, Genes Dev. 20, 990 (2006).

[72] N. G. Van Kampen, Stochastic Processes in Physics and Chemistry (Elsevier, North Holland, 1992).

[73] D. T. Gillespie, A General Method for Numerically Simulating the Stochastic Time Evolution of Coupled Chemical Reactions, J. Comput. Phys. 22, 403 (1976).

[74] L. E. Wybenga-Groot, C. S. Ho, F. D. Sweeney, D. F. Ceccarelli, C. J. McGlade, D. Durocher, and F. Sicheri, Structural Basis of Rad53 Kinase Activation by Dimerization and Activation Segment Exchange, Cell. Signalling 26, 1825 (2014).

[75] E. S. Chen, J. H. Weng, Y. H. Chen, S. C. Wang, X. X. Liu, W. C. Huang, T. Matsui, Y. Kawano, J. H. Liao, L. H. Lim, Y. Bessho, K. F. Huang, W. J. Wu, and M. D. Tsai, Phosphopriming Confers Functionally Relevant Specificities for Rad53 Kinase Autophosphorylation, Biochemistry 56, 5112 (2017). 